\title{
A new Family of Main Chain Polymeric Lewis Acids: Synthesis and Fluorescent Sensing Properties of Boron-modified Polythiophenes
}

\author{
Anand Sundararaman, Maria Victor, Resmi Varughese, and Frieder Jäkle* \\ Department of Chemistry, Rutgers University-Newark, 73 Warren Street, Newark, NJ 07102
}

\section{Supplementary Material}

Materials and General Methods. Pyridine (anhydrous grade), bromine, and ${ }^{\mathrm{n}} \mathrm{BuLi}$ (1.6M in hexanes), were purchased from Acros, trimethyltin chloride, and 1,2bis(diphenylphosphino)ethane nickel(II) chloride from Strem Chemicals, $\mathrm{BBr}_{3}$ from Aldrich, $\mathrm{C}_{6} \mathrm{~F}_{5} \mathrm{Br}$ from Fluorochem, 4-bromocumene from Lancaster, and 3-bromothiophene from Oakwood products. $\mathrm{BBr}_{3}$ and $\mathrm{C}_{6} \mathrm{~F}_{5} \mathrm{Br}$ were distilled under vacuum and 3-bromothiophene was distilled from $\mathrm{CaH}_{2}$ prior to use. All other chemicals were used as received without further purification. 5,5'Bis(trimethylstannyl)-3,3'-dihexyl-2,2'-bithiophene ${ }^{[1]}, \mathrm{C}_{6} \mathrm{~F}_{5} \mathrm{BBr}_{2}{ }^{[2]}$, 4-trimethylstannylcumene ${ }^{[3]}$, dibromoborylcumene $^{[3]}$, 2-trimethyl-stannylthiophene ${ }^{[3]}$, and 2-dibromoborylthiophene ${ }^{[3]}$, dibromoborylferrocene ${ }^{[4]}$, were prepared according to literature procedures. All reactions and manipulations were carried out under an atmosphere of prepurified nitrogen using either Schlenk techniques or an inert-atmosphere glove box (Innovative Technologies). Ether solvents were distilled from $\mathrm{Na}$ /benzophenone prior to use. Hydrocarbon and chlorinated solvents were purified using a solvent purification system (Innovative Technologies; alumina / copper columns for hydrocarbon solvents), and the chlorinated solvents were subsequently distilled from $\mathrm{CaH}_{2}$ and degassed via several freeze-pump-thaw cycles.

All $499.893 \mathrm{MHz}{ }^{1} \mathrm{H}, 125.7 \mathrm{MHz}{ }^{13} \mathrm{C}, 160.4 \mathrm{MHz}{ }^{11} \mathrm{~B}$ NMR, and 470.2 $\mathrm{MHz}{ }^{19} \mathrm{~F}$ NMR spectra were recorded on a Varian INOVA spectrometer equipped with a boron-free $5 \mathrm{~mm}$ dual broadband gradient probe (Nalorac, Varian Inc., Martinez, CA). Solution ${ }^{1} \mathrm{H}$ and

${ }^{13} \mathrm{C}$ NMR spectra were referenced internally to solvent signals. ${ }^{11} \mathrm{~B}$ NMR spectra were referenced externally to $\mathrm{BF}_{3} \cdot \mathrm{Et}_{2} \mathrm{O}(\square=0)$ and ${ }^{19} \mathrm{~F}$ NMR spectra were referenced to $\square, \square$ ', $\square$ '-trifluorotoluene $(0.05 \%$ in $\left.\mathrm{C}_{6} \mathrm{D}_{6} ; \square=-67.73\right) .{ }^{11} \mathrm{~B}$ NMR spectra were acquired with boron-free quartz NMR tubes. All NMR spectra were recorded at ambient temperature unless noted otherwise. The abbreviations Th (2-thienyl), $\mathrm{Hx}_{2} \mathrm{Th}_{2}$ (3,3'-dihexyl-2,2'-bithiophenediyl), Ph (phenyl), Pf (2,3,4,5,6-pentafluorophenyl), Fc (ferrocenyl), and Hx (n-hexyl) are used for the peak assignments.

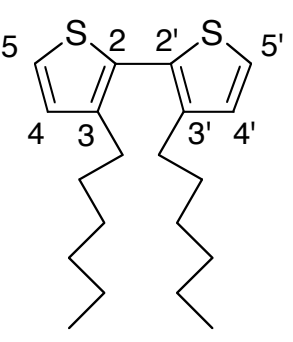

$\mathrm{Hx}_{2} \mathrm{Th}_{2}$<smiles>CC(C)c1ccc(P)cc1</smiles>

i $\mathrm{PrPh}$

UV-visible absorption data were acquired on a Varian Cary 500 UV-Vis / NIR spectrophotometer. The fluorescence data and quantum yields were measured on a Varian Cary Eclipse Fluorescence spectrophotometer with the same solutions as those used in the UV-visible measurements. Anthracene was used as the standard for determination of the quantum yields ( $\square$ ). The quantum yield of anthracene was adopted from the 'Handbook of Photochemistry, $2^{\text {nd }}$ Edition, Revised and Expanded. ISBN 0-8247-7911-8' and the concentration of anthracene in THF was 
$6.86 \cdot 10^{-6} \mathrm{M}$. Sample solutions and pyridine solutions were prepared using a microbalance $( \pm 0.1$ $\mathrm{mg}$ ) and volumetric glassware. Titrations experiments were performed by successive addition of of predetermined amounts of the pyridine solution.

GPC analyses were performed in toluene containing $2 \%$ pyridine $(1 \mathrm{ml} / \mathrm{min})$ using a Waters Breeze system equipped with a 717plus autosampler, a 1525 binary HPLC pump, a 2487 dual $\square$ absorbance detector, and a 2414 refractive index detector. For separation the samples were passed through a series of styragel columns (Polymer Laboratories; two columns $5 \square \mathrm{m} / 100 \AA$ and one column $5 \square \mathrm{m} / 500 \AA$ in series), which were kept in a column heater at $35^{\circ} \mathrm{C}$. The columns were calibrated with polystyrene standards (Polymer Laboratories).

MALDI-TOF-TOF measurements were performed on an Applied Biosystems 4700 Proteomics Analyzer in reflective mode with delayed extraction. Benzo[a]pyrene was used as the matrix (10 $\mathrm{mg} / \mathrm{ml}$ toluene $).{ }^{[5]}$ Samples were prepared in toluene $(10 \mathrm{mg} / \mathrm{ml})$, mixed with the matrix in a 1:10 ratio, and then spotted on the wells of a sample plate inside a glove box. Ferrocene substituted polymers and model compounds were measured in positive mode; iso-propylphenyl and pentafluorophenyl substituted polymers and model compounds were observed in negative mode. Peptides were used for calibration in the positive mode (Des-Arg-Bradykinin (904.4681), Angiotensin I (1296.6853), Glu-Fibrinopeptide B (1570.6774), ACTH (clip 1-17) (2093.0867), ACTH (clip 18-39) (2465.1989), ACTH (clip 7-38) (3657.9294) with $\square$-hydroxy-4-cyanocinnamic acid as the matrix). The model compounds M-BPf and M-BPh served as calibrants for the polymers PTh-BPh and PTh-BPf in negative mode.

Caution! $\mathrm{BBr}_{3}$ is toxic and highly corrosive and should be handled appropriately with great care. Fluorinated grease was used for ground glass joints in reactions involving $\mathrm{BBr}_{3}$.

\section{Synthesis of Organoborane Polymers PTh-BAr}

Polycondensation of $\mathbf{F c B B r}_{2}$ and $\mathbf{T h}_{2} \mathbf{H x}_{2}\left(\mathbf{S n M e}_{3}\right)_{2}: \mathrm{FcBBr}_{2}(2.00 \mathrm{~g}, 5.62 \mathrm{mmol})$ was dissolved in $20 \mathrm{ml}$ of $\mathrm{CH}_{2} \mathrm{Cl}_{2}$ and cooled to $-35^{\circ} \mathrm{C}$ under stirring. A solution of $\mathrm{Th}_{2} \mathrm{Hx}_{2}\left(\mathrm{SnMe}_{3}\right)_{2}(3.72 \mathrm{~g}, 5.62$ mmol) in $30 \mathrm{ml}$ of $\mathrm{CH}_{2} \mathrm{Cl}_{2}$ was added slowly under stirring. After addition the reaction mixture was allowed to warm up slowly and left stirring at room temperature for $24 \mathrm{~h}$. The reaction solution was concentrated to $2 \mathrm{ml}$ under high vacuum and precipitated into $500 \mathrm{ml}$ of cold hexanes to give a dark red powder. The product was dried overnight at $60{ }^{\circ} \mathrm{C}$ under high vacuum. Isolated yield: 1.7 g (57\%). For PTh-BFc: ${ }^{1} \mathrm{H} \mathrm{NMR}\left(\mathrm{CDCl}_{3}, 499.893 \mathrm{MHz}\right): \square=7.71\left(\mathrm{~s}, 2 \mathrm{H}, \mathrm{Hx}_{2} \mathrm{Th}_{2^{-}}\right.$ H4,4'), 4.94 (b, 2H, Cp-H), 4.82 (b, 2H, Cp-H), 4.25 (s, 5H, $\left.\mathrm{C}_{5} \mathrm{H}_{5}\right), 2.80$ (t, ${ }^{3} \mathrm{~J}=8.0 \mathrm{~Hz}, 4 \mathrm{H}$, Th$\left.\mathrm{CH}_{2}\right), 1.74\left(\mathrm{~m}, 4 \mathrm{H}\right.$, hexyl-H), 1.4-1.3 (m, 12H, hexyl-H), $0.87\left(\mathrm{~m}, 6 \mathrm{H}, \mathrm{CH}_{3}\right)$; end groups: $7.35(\mathrm{~d}$, ${ }^{3} \mathrm{~J}=5.0 \mathrm{~Hz}, \mathrm{Hx}_{2} \mathrm{Th}_{2}-\mathrm{H} 5$ '), 7.03 (d, $\left.{ }^{3} \mathrm{~J}=5.0 \mathrm{~Hz}, \mathrm{Hx}_{2} \mathrm{Th}_{2}-\mathrm{H} 4{ }^{\prime}\right) ;{ }^{13} \mathrm{C} \mathrm{NMR}\left(\mathrm{CDCl}_{3}, 125.7 \mathrm{MHz}\right): \square=$ 144.8, 143.4, 141.7, 138.4 $\left(\mathrm{Hx}_{2} \mathrm{Th}_{2}\right)$, 78.2, 75.5, $74.1(\mathrm{Cp}-\mathrm{C}), 70.1\left(\mathrm{C}_{5} \mathrm{H}_{5}\right), 31.9,31.3,29.5,29.4$, 22.9, 14.3 (hexyl-C); ${ }^{11} \mathrm{~B}$ NMR $\left(\mathrm{CDCl}_{3}, 160.3 \mathrm{MHz}\right)$ : $\square=48.7\left(\mathrm{w}_{1 / 2}=4000 \mathrm{~Hz}\right) ; \mathrm{UV}-\mathrm{V}$ is $\left(\mathrm{CH}_{2} \mathrm{Cl}_{2}\right)$ : $\square_{\max }=379 \mathrm{~nm}(\square=13600), 497 \mathrm{~nm}(\square=2770)$; $\mathrm{TGA}\left(\mathrm{N}_{2}, 20{ }^{\circ} \mathrm{C} / \mathrm{min}\right): T_{\mathrm{dec}}=112{ }^{\circ} \mathrm{C}$ (onset, $92 \%$ multi-step weight at $500{ }^{\circ} \mathrm{C}$ ).

Polycondensation of ${ }^{\mathrm{i}} \mathrm{PrPhBBr}_{2}$ and $\mathbf{T h}_{\mathbf{2}} \mathbf{H x}_{\mathbf{2}}\left(\mathbf{S n M e}_{3}\right)_{2}$ : A solution of ${ }^{\mathrm{i}} \mathrm{PrPhBBr}_{2}(2.97 \mathrm{~g}, 10.26$ mmol) in $50 \mathrm{ml}$ of $\mathrm{CH}_{2} \mathrm{Cl}_{2}$ was cooled to $-35{ }^{\circ} \mathrm{C}$. $\mathrm{Th}_{2} \mathrm{Hx}_{2}\left(\mathrm{SnMe}_{3}\right)_{2}(6.77 \mathrm{~g}, 10.26 \mathrm{mmol})$ was dissolved in $50 \mathrm{ml}$ of $\mathrm{CH}_{2} \mathrm{Cl}_{2}$ and added slowly under stirring. The mixture was allowed to warm up slowly and left stirring at room temperature for $24 \mathrm{~h}$. The reaction solution was concentrated to $2 \mathrm{ml}$ under high vacuum and precipitated into $500 \mathrm{ml}$ of cold hexanes. The bright yellow powdery 
product was dried overnight at $60{ }^{\circ} \mathrm{C}$ under high vacuum. Isolated yield: $3.2 \mathrm{~g}(67 \%)$. For PTh-BPh: ${ }^{1} \mathrm{H}$ NMR $\left(\mathrm{CDCl}_{3}, 499.893 \mathrm{MHz}\right): \mathrm{C}=7.80\left(\mathrm{~d},{ }^{3} \mathrm{~J}=8.0 \mathrm{~Hz}, 2 \mathrm{H}, \mathrm{Ph}-\mathrm{H}_{o}\right), 7.71(\mathrm{~s}, 2 \mathrm{H}$, $\left.\mathrm{Hx}_{2} \mathrm{Th}_{2}-\mathrm{H} 4,4^{\prime}\right), 7.35\left(\mathrm{~d},{ }^{3} \mathrm{~J}=8.0 \mathrm{~Hz}, 2 \mathrm{H}, \mathrm{Ph}-\mathrm{H}_{m}\right), 3.01$ (heptet, $\left.{ }^{3} \mathrm{~J}=7.0 \mathrm{~Hz}, 1 \mathrm{H}, \mathrm{CH}\left[\mathrm{CH}_{3}\right]_{2}\right), 2.71$ (m, 4H, Th- $\left.\mathrm{CH}_{2}\right), 1.63\left(\mathrm{~m}, 4 \mathrm{H}\right.$, hexyl-H), $1.34\left(\mathrm{~d},{ }^{3} \mathrm{~J}=7.0 \mathrm{~Hz}, 6 \mathrm{H}, \mathrm{CH}\left[\mathrm{CH}_{3}\right]_{2}\right), 1.30-1.20(\mathrm{~m}, 12 \mathrm{H}$, hexyl- $\mathrm{H}), 0.83\left(\mathrm{~m}, 6 \mathrm{H}, \mathrm{CH}_{3}\right)$; end groups: $6.99\left(\mathrm{~d},{ }^{3} \mathrm{~J}=5.0 \mathrm{~Hz}, \mathrm{Hx}_{2} \mathrm{Th}_{2}-\mathrm{H}_{4}{ }^{\prime}\right), \mathrm{Hx}_{2} \mathrm{Th}_{2}-\mathrm{H} 5$ ' was not resolved; ${ }^{13} \mathrm{C} \mathrm{NMR}\left(\mathrm{CDCl}_{3}, 125.7 \mathrm{MHz}\right): \mathrm{Q}=151.8\left(\mathrm{Ph}-\mathrm{C}_{\mathrm{p}}\right), 145.1,144.5,144.3,141.8\left(\mathrm{Hx}_{2} \mathrm{Th}_{2}\right)$, $140.7\left(\mathrm{Ph}-\mathrm{C}_{i}\right), 137.4\left(\mathrm{Ph}-\mathrm{C}_{o}\right), 125.8\left(\mathrm{Ph}-\mathrm{C}_{m}\right), 34.4\left(\mathrm{CH}_{[}\left[\mathrm{CH}_{3}\right]_{2}\right), 24.1\left(\mathrm{CH}\left[\mathrm{CH}_{3}\right]_{2}\right), 31.9,31.2,29.4$, 29.2, 22.8, 14.3 (hexyl-C); ${ }^{11} \mathrm{~B}$ NMR $\left(\mathrm{CDCl}_{3}, 160.3 \mathrm{MHz}\right): \square=46.2\left(\mathrm{w}_{1 / 2}=5600 \mathrm{~Hz}\right)$; UV-Vis $\left(\mathrm{CH}_{2} \mathrm{Cl}_{2}\right): \square_{\max }=391 \mathrm{~nm}(\square=19690)$, fluorescence $\left(\mathrm{CH}_{2} \mathrm{Cl}_{2}\right): \square_{\mathrm{em}, \max }=491 \mathrm{~nm}, \mathrm{\square}=0.21\left(\square_{\mathrm{exc}}=\right.$ $390 \mathrm{~nm}$ ); TGA $\left(\mathrm{N}_{2}, 20{ }^{\circ} \mathrm{C} / \mathrm{min}\right.$ ): $T_{\mathrm{dec}}=210{ }^{\circ} \mathrm{C}$ (onset, $90 \%$ single step weight loss at $300{ }^{\circ} \mathrm{C}$ ).

Polycondensation of $\mathbf{P f B B r}_{2}$ and $\mathbf{T h}_{2} \mathbf{H x}_{2}\left(\mathbf{S n M e}_{3}\right)_{2}: \mathrm{Th}_{2} \mathrm{Hx}_{2}\left(\mathrm{SnMe}_{3}\right)_{2}(0.98 \mathrm{~g}, 1.48 \mathrm{mmol})$ was dissolved in $20 \mathrm{ml}$ of $\mathrm{CH}_{2} \mathrm{Cl}_{2}$ and cooled to $-35{ }^{\circ} \mathrm{C}$ under stirring. A solution of $\mathrm{PfBBr}_{2}(0.50 \mathrm{~g}$, $1.48 \mathrm{mmol})$ in $20 \mathrm{ml}$ of $\mathrm{CH}_{2} \mathrm{Cl}_{2}$ was added slowly under stirring. After addition the reaction mixture was allowed to warm up slowly and left stirring at room temperature for $24 \mathrm{~h}$. The solvent was removed under high vacuum to obtain a fluorescent yellow oily material. The crude product was heated to $60{ }^{\circ} \mathrm{C}$ for $6 \mathrm{~h}$ to remove trimethyltin bromide. The fluorescent yellow product was used without further purification. Isolated yield: $0.54 \mathrm{~g}(71 \%)$. For PTh-BPf: ${ }^{1} \mathrm{H}$ NMR $\left(\mathrm{CDCl}_{3}\right.$, $499.893 \mathrm{MHz}$ ): $\mathrm{C}=7.67$ (s, 2H, $\left.\mathrm{Hx}_{2} \mathrm{Th}_{2}-\mathrm{H} 4,4^{\prime}\right), 2.64\left(\mathrm{t},{ }^{3} \mathrm{~J}=7.5 \mathrm{~Hz}, 4 \mathrm{H}, \mathrm{Th}^{-} \mathrm{CH}_{2}\right), 1.57(\mathrm{~m}, 4 \mathrm{H}$, hexyl-H), 1.3-1.2 (m, $12 \mathrm{H}$, hexyl-H), $0.81\left(\mathrm{~m}, 6 \mathrm{H}, \mathrm{CH}_{3}\right)$; end groups: $7.36\left(\mathrm{~d},{ }^{3} \mathrm{~J}=5.0 \mathrm{~Hz}, \mathrm{Hx}_{2} \mathrm{Th}_{2}{ }^{-}\right.$ $\left.\mathrm{H} 5^{\prime}\right), 7.00\left(\mathrm{~d},{ }^{3} \mathrm{~J}=5.0 \mathrm{~Hz}, \mathrm{Hx}_{2} \mathrm{Th}_{2}-\mathrm{H} 4^{\prime}\right) ;{ }^{19} \mathrm{~F}$ NMR $\left(470.2 \mathrm{MHz}, \mathrm{CDCl}_{3}, 20{ }^{\circ} \mathrm{C}\right)$ : $\mathrm{C}=-130.9(\mathrm{~m}, 2 \mathrm{~F}$, Pf- $\left.\mathrm{F}_{\mathrm{o}}\right),-154.1\left(\mathrm{~m}, 1 \mathrm{~F}, \mathrm{Pf}-\mathrm{F}_{\mathrm{p}}\right),-162.7$ (m, 2F, Pf- $\left.\mathrm{F}_{\mathrm{m}}\right) ;{ }^{13} \mathrm{C} \mathrm{NMR}\left(\mathrm{CDCl}_{3}, 125.7 \mathrm{MHz}\right): \mathrm{C}=145.8$, 145.5 $\left(\mathrm{Hx}_{2} \mathrm{Th}_{2}\right), 146.0\left(\mathrm{dm},{ }^{1} \mathrm{~J}_{\mathrm{CF}}=244 \mathrm{~Hz}, \mathrm{Pf}-\mathrm{C}_{\mathrm{o}}\right), 143.8\left(\mathrm{Hx}_{2} \mathrm{Th}_{2}\right), 141.7\left(\mathrm{dm},{ }^{1} \mathrm{~J}_{\mathrm{CF}}=251 \mathrm{~Hz}, \mathrm{Pf}-\right.$ $\left.\mathrm{C}_{\mathrm{p}}\right), 137.5\left(\mathrm{dm},{ }^{1} \mathrm{~J}_{\mathrm{CF}}=251 \mathrm{~Hz}, \mathrm{Pf}-\mathrm{C}_{\mathrm{m}}\right), 142.8\left(\mathrm{Hx}_{2} \mathrm{Th}_{2}\right), 114.9\left(\mathrm{t},{ }^{3} \mathrm{~J}_{\mathrm{FF}}=30 \mathrm{~Hz}, \mathrm{Pf}-\mathrm{C}_{\mathrm{i}}\right), 31.7,31.3$, 29.5, 29.4, 22.7, 14.2 (hexyl-C); ${ }^{11} \mathrm{~B}$ NMR $\left(\mathrm{CDCl}_{3}, 160.3 \mathrm{MHz}\right)$ : $\square=46\left(\mathrm{w}_{1 / 2}=3500 \mathrm{~Hz}\right) ; \mathrm{UV}-\mathrm{Vis}$ $\left(\mathrm{CH}_{2} \mathrm{Cl}_{2}\right): \square_{\max }=413 \mathrm{~nm}(\square=17820)$; fluorescence $\left(\mathrm{CH}_{2} \mathrm{Cl}_{2}\right): \square_{\mathrm{em} \text {,max }}=529, \square=0.15\left(\square_{\mathrm{exc}}=415\right.$ $\mathrm{nm}$ ); TGA $\left(\mathrm{N}_{2}, 20^{\circ} \mathrm{C} / \mathrm{min}\right)$ : $T_{\mathrm{dec}}=222{ }^{\circ} \mathrm{C}$ (onset, $93 \%$ single step weight loss at $300^{\circ} \mathrm{C}$ ).

\section{Pyridine Binding to Polymers PTh-BAr}

Pyridine Binding to PTh-BFc: A solution of pyridine (75 mg, $0.95 \mathrm{mmol}$ ) in $10 \mathrm{ml}$ of $\mathrm{CH}_{2} \mathrm{Cl}_{2}$ was added dropwise with stirring to a solution of PTh-BFc $(200 \mathrm{mg}, 0.378 \mathrm{mmol})$ in $10 \mathrm{ml}$ of $\mathrm{CH}_{2} \mathrm{Cl}_{2}$. The color changed from red to pale yellow. The reaction mixture was kept stirring for $5 \mathrm{~h}$. The solvent was removed under high vacuum and the crude product was washed with hexanes several times. The orange powdery product was dried overnight under high vacuum. Isolated yield: $172 \mathrm{mg}$ (75 \%). For PTh-BFc•Py: ${ }^{1} \mathrm{H}$ NMR $\left(\mathrm{CDCl}_{3}, 499.893 \mathrm{MHz}\right)$ : $\square=8.74$ (b, 2H, Py$\left.\mathrm{H}_{\mathrm{o}}\right), 7.94\left(\mathrm{t},{ }^{3} \mathrm{~J}=7.0 \mathrm{~Hz}, 1 \mathrm{H}, \mathrm{Py}-\mathrm{H}_{\mathrm{p}}\right), 7.48\left(\mathrm{~b}, 2 \mathrm{H}, \mathrm{Py}-\mathrm{H}_{\mathrm{m}}\right), 6.87\left(\mathrm{~s}, 2 \mathrm{H}, \mathrm{Hx}_{2} \mathrm{Th}_{2}-\mathrm{H} 4,4\right.$ '), 4.30 (b, 2H, Cp-H), 4.27 (b, 2H, Cp-H), 4.05 (s, 5H, $\mathrm{C}_{5} \mathrm{H}_{5}$ ), 2.64 (b, 4H, Th- $\mathrm{CH}_{2}$ ), 1.60 (bm, 4H, hexyl-H), 1.4$1.3\left(\mathrm{bm}, 12 \mathrm{H}\right.$, hexyl-H), $0.85\left(\mathrm{~b}, 6 \mathrm{H}, \mathrm{CH}_{3}\right)$; end groups: $7.25\left(\mathrm{~d},{ }^{3} \mathrm{~J}=5.0 \mathrm{~Hz}, \mathrm{Hx}_{2} \mathrm{Th}_{2}-\mathrm{H} 5\right.$ '), $6.98(\mathrm{~d}$, $\left.{ }^{3} \mathrm{~J}=5.0 \mathrm{~Hz}, \mathrm{Hx}_{2} \mathrm{Th}_{2}-\mathrm{H} 4{ }^{\prime}\right) ;{ }^{13} \mathrm{C} \mathrm{NMR}\left(\mathrm{CDCl}_{3}, 125.7 \mathrm{MHz}\right): \mathrm{\square}=153.3,147.5,141.3,140.1,133.5$, 131.9, $125.0\left(\mathrm{Hx}_{2} \mathrm{Th}_{2}\right.$ and Py $), 86.8\left(\mathrm{Cp}-\mathrm{C}_{\mathrm{i}}\right), 74.7,69.6(\mathrm{Cp}-\mathrm{C}), 69.0\left(\mathrm{C}_{5} \mathrm{H}_{5}\right), 31.9,31.3,29.5,29.4$, 22.9, 14.3 (hexyl-C); ${ }^{11} \mathrm{~B}$ NMR $\left(\mathrm{CD}_{2} \mathrm{Cl}_{2}, 160.3 \mathrm{MHz}\right): \square=1.4\left(\mathrm{w}_{1 / 2}=1600 \mathrm{~Hz}\right) ; \mathrm{GPC} / \mathrm{RI}$ (in toluene with $2 \%$ pyridine against PS standards) $M_{\mathrm{n}}=5920, P D I=1.15 ; \mathrm{UV}-\mathrm{Vis}\left(\mathrm{CH}_{2} \mathrm{Cl}_{2}\right)$ : $\square_{\max }=$ $374 \mathrm{~nm}(\square=9590), 490 \mathrm{~nm}(\square=1050)$. 
Pyridine Binding to PTh-BPh: A solution of pyridine (75 mg, $0.95 \mathrm{mmol}$ ) in $10 \mathrm{ml}$ of $\mathrm{CH}_{2} \mathrm{Cl}_{2}$ was added dropwise under stirring to a solution of PTh-BPh $(200 \mathrm{mg}, 0.432 \mathrm{mmol})$ in $10 \mathrm{ml}$ of $\mathrm{CH}_{2} \mathrm{Cl}_{2}$. The color changed immediately from fluorescent yellow to pale yellow. The reaction mixture was kept stirring for $5 \mathrm{~h}$. The solvent was removed under high vacuum, and the crude product was washed with hexanes several times. The colorless powdery product was dried overnight under high vacuum. Isolated yield: $150 \mathrm{mg}(64 \%)$. For PTh-BPh•Py: ${ }^{1} \mathrm{H}$ NMR $\left(\mathrm{CDCl}_{3}\right.$, $499.893 \mathrm{MHz}): \mathrm{\square}=8.62\left(\mathrm{~d},{ }^{3} \mathrm{~J}=5.5 \mathrm{~Hz}, 2 \mathrm{H}, \mathrm{Py}-\mathrm{H}_{\mathrm{o}}\right), 7.98\left(\mathrm{t},{ }^{3} \mathrm{~J}=7.5 \mathrm{~Hz}, 1 \mathrm{H}, \mathrm{Py}-\mathrm{H}_{\mathrm{p}}\right), 7.49\left(\mathrm{pst},{ }^{3} \mathrm{~J}\right.$ $\left.=7.0 \mathrm{~Hz}, 2 \mathrm{H}, \mathrm{Py}-\mathrm{H}_{\mathrm{m}}\right), 7.16\left(\mathrm{~d},{ }^{3} \mathrm{~J}=8.0 \mathrm{~Hz}, 2 \mathrm{H}, \mathrm{Ph}-\mathrm{H}_{o}\right), 7.10\left(\mathrm{~d},{ }^{3} \mathrm{~J}=8.0 \mathrm{~Hz}, 2 \mathrm{H}, \mathrm{Ph}-\mathrm{H}_{m}\right), 6.93(\mathrm{~s}$, $2 \mathrm{H}, \mathrm{Hx}_{2} \mathrm{Th}_{2}-\mathrm{H} 4,4$ '), 2.89 (heptet, ${ }^{3} \mathrm{~J}=7.0 \mathrm{~Hz}, 1 \mathrm{H}, \mathrm{CH}\left[\mathrm{CH}_{3}\right]_{2}$ ), 2.50 (t, ${ }^{3} \mathrm{~J}=6.5 \mathrm{~Hz}, 2 \mathrm{H}$, Th- $\mathrm{CH}_{2}$ ), $1.51\left(\mathrm{~b}, 2 \mathrm{H}\right.$, hexyl-H), $1.26\left(\mathrm{~d},{ }^{3} \mathrm{~J}=7.0 \mathrm{~Hz}, 6 \mathrm{H}, \mathrm{CH}\left[\mathrm{CH}_{3}\right]_{2}\right), 1.24\left(\mathrm{~m}, 12 \mathrm{H}\right.$, hexyl-H), $0.84\left(\mathrm{t},{ }^{3} \mathrm{~J}=\right.$ $\left.7.0 \mathrm{~Hz}, 6 \mathrm{H}, \mathrm{CH}_{3}\right)$; end groups: $7.21\left(\mathrm{~d},{ }^{3} \mathrm{~J}=5.0 \mathrm{~Hz}, \mathrm{Hx}_{2} \mathrm{Th}_{2}-\mathrm{H} 5\right.$ '), $\mathrm{Hx}_{2} \mathrm{Th}_{2}-\mathrm{H} 4$ ' not resolved; ${ }^{13} \mathrm{C}$ NMR $\left(\mathrm{CDCl}_{3}, 125.7 \mathrm{MHz}\right): \mathrm{C}=153.1,148.7,147.6,146.1,141.8,140.5,134.7,133.7,132.5$, 125.3, $124.9\left(\mathrm{Hx}_{2} \mathrm{Th}_{2}, \mathrm{Ph}\right.$ and Py), $33.9\left(\mathrm{CH}\left[\mathrm{CH}_{3}\right]_{2}\right), 24.3\left(\mathrm{CH}\left[\mathrm{CH}_{3}\right]_{2}\right), 31.9,31.1,29.5,29.3,22.9$, 14.3 (hexyl-C); ${ }^{11} \mathrm{~B}$ NMR $\left(\mathrm{CDCl}_{3}, 160.3 \mathrm{MHz}\right):-\mathrm{C}=-0.7\left(\mathrm{w}_{1 / 2}=1400 \mathrm{~Hz}\right)$; GPC/RI (in toluene with $2 \%$ pyridine against $\mathrm{PS}$ standards) $M_{\mathrm{n}}=9350, P D I=1.19$.

Pyridine Binding to PTh-BPf: A solution of pyridine $(50 \mathrm{mg}, 0.63 \mathrm{mmol})$ in $10 \mathrm{ml}$ of $\mathrm{CH}_{2} \mathrm{Cl}_{2}$ was added dropwise to a solution of PTh-BPf (200 mg, $0.39 \mathrm{mmol}$ ) in $10 \mathrm{ml}$ of $\mathrm{CH}_{2} \mathrm{Cl}_{2}$. The color changed immediately from fluorescent yellow to pale yellow. The reaction mixture was kept stirring for $5 \mathrm{~h}$. The solvent was removed under high vacuum, and the crude product was washed with hexanes several times. The faint yellow material was dried overnight under high vacuum. Isolated yield: $140 \mathrm{mg}(60 \%)$. For PTh-BPf•Py: ${ }^{1} \mathrm{H}$ NMR $\left(\mathrm{CDCl}_{3}, 499.893 \mathrm{MHz}\right): \square=8.60$ (b, 2H, Py- $\mathrm{H}_{\mathrm{o}}$ ), 8.01 (b, 1H, Py- $\mathrm{H}_{\mathrm{p}}$ ), 7.51 (b, 2H, Py- $\mathrm{H}_{\mathrm{m}}$ ), 6.97 (s, 2H, $\mathrm{Hx}_{2} \mathrm{Th}_{2}-\mathrm{H} 4,4$ '), 2.50 (b, 4H, Th$\left.\mathrm{CH}_{2}\right), 1.50\left(\mathrm{~b}, 4 \mathrm{H}\right.$, hexyl-H), $1.20(\mathrm{~b}, 12 \mathrm{H}$, hexyl- $\mathrm{H}), 0.81\left(\mathrm{~b}, 6 \mathrm{H}, \mathrm{CH}_{3}\right)$; end groups: $7.22\left(\mathrm{~d},{ }^{3} \mathrm{~J}=\right.$ $5.0 \mathrm{~Hz}, \mathrm{Hx}_{2} \mathrm{Th}_{2}-\mathrm{H} 5$ '), 6.93 (d, ${ }^{3} \mathrm{~J}=5.0 \mathrm{~Hz}, \mathrm{Hx}_{2} \mathrm{Th}_{2}-\mathrm{H} 4$ '); ${ }^{19} \mathrm{~F} \mathrm{NMR}\left(470.2 \mathrm{MHz}, \mathrm{CDCl}_{3}, 20{ }^{\circ} \mathrm{C}\right)$ : $\mathrm{C}=-130.9$ (b, 2F, Pf- $\left.\mathrm{F}_{\mathrm{o}}\right),-159.6$ (b, 1F, Pf- $\left.\mathrm{F}_{\mathrm{p}}\right),-165.1$ (b, 2F, Pf- $\left.\mathrm{F}_{\mathrm{m}}\right) ;{ }^{13} \mathrm{C}$ NMR $\left(\mathrm{CDCl}_{3}, 125.7\right.$ $\mathrm{MHz}): \mathrm{C}=149.7,147.4,142.3,141.4,134.6,132.5,125.4\left(\mathrm{Hx}_{2} \mathrm{Th}_{2}\right.$ and $\left.\mathrm{Py}\right), 148.1\left(\mathrm{dm},{ }^{1} \mathrm{~J}_{\mathrm{CF}}=241\right.$ $\left.\mathrm{Hz}, \mathrm{Pf}_{-} \mathrm{C}_{\mathrm{o}}\right), 137.5\left(\mathrm{dm},{ }^{1} \mathrm{~J}_{\mathrm{CF}}=245 \mathrm{~Hz}, \mathrm{Pf}-\mathrm{C}_{\mathrm{m}}\right), 139.9\left(\mathrm{dm},{ }^{1} \mathrm{~J}_{\mathrm{CF}}=250 \mathrm{~Hz}, \mathrm{Pf}-\mathrm{C}_{\mathrm{p}}\right), 121.7\left(\mathrm{~b}, \mathrm{Pf}_{-} \mathrm{C}_{\mathrm{i}}\right)$, 31.8, 31.0, 29.4, 29.3, 22.8, 14.2 (hexyl-C); ${ }^{11} \mathrm{~B}$ NMR $\left(\mathrm{CDCl}_{3}, 160.3 \mathrm{MHz}\right): \square=-3.1\left(\mathrm{w}_{1 / 2}=960\right.$ $\mathrm{Hz}$ ); GPC/RI (in toluene with $2 \%$ pyridine against PS standards) $M_{\mathrm{n}}=5280, P D I=1.48$.

\section{Synthesis of Model Compounds:}

Reaction of $\mathbf{T h S n M e}_{3}$ with $\mathrm{FcBBr}_{2}$ : Synthesis of FcThBBr. A solution of $\mathrm{ThSnMe}_{3}(1.39 \mathrm{~g}$, $5.62 \mathrm{mmol})$ in $10 \mathrm{ml}$ of $\mathrm{CH}_{2} \mathrm{Cl}_{2}$ was added slowly under stirring to a solution of $\mathrm{FcBBr}_{2}(2.00 \mathrm{~g}$, $5.62 \mathrm{mmol}$ ) in $10 \mathrm{ml}$ of $\mathrm{CH}_{2} \mathrm{Cl}_{2}$ at $-35{ }^{\circ} \mathrm{C}$. The reaction mixture was allowed to warm up slowly and left stirring at room temperature for $12 \mathrm{~h}$. All volatile components were removed under high vacuum and the crude product was heated to $60{ }^{\circ} \mathrm{C}$ for $6 \mathrm{~h}$ to remove trimethyltin bromide. The dark red crude material was recrystallized from hexanes at $-35{ }^{\circ} \mathrm{C}$. Isolated yield: $1.77 \mathrm{~g}(88 \%)$. For FcThBBr: ${ }^{1} \mathrm{H}$ NMR $\left(\mathrm{CDCl}_{3}, 499.893 \mathrm{MHz}\right): \square=8.08$ (d, $\left.{ }^{3} \mathrm{~J}=3.5 \mathrm{~Hz}, 1 \mathrm{H}, \mathrm{Th}-\mathrm{H} 3\right), 7.90\left(\mathrm{~d},{ }^{3} \mathrm{~J}\right.$ $=4.5 \mathrm{~Hz}, 1 \mathrm{H}, \mathrm{Th}-\mathrm{H} 5), 7.30\left(\mathrm{dd},{ }^{3} \mathrm{~J}=3.5 / 4.5 \mathrm{~Hz}, 1 \mathrm{H}, \mathrm{Th}-\mathrm{H} 4\right), 4.88\left(\mathrm{pst},{ }^{3 / 4} \mathrm{~J}=1.5 \mathrm{~Hz}, 2 \mathrm{H}, \mathrm{Cp}-\mathrm{H}\right)$, 4.85 (pst, $\left.{ }^{3 / 4} \mathrm{~J}=1.5 \mathrm{~Hz}, 2 \mathrm{H}, \mathrm{Cp}-\mathrm{H}\right), 4.16\left(\mathrm{~s}, 5 \mathrm{H}, \mathrm{C}_{5} \mathrm{H}_{5}\right) ;{ }^{13} \mathrm{C} \mathrm{NMR}\left(\mathrm{CDCl}_{3}, 125.7 \mathrm{MHz}\right): \square=141.1$, 136.1 (Th-C3), (Th-C5), 129.0 (Th-C4), 77.6 (Cp-C), second Cp-C signal overlaps with $\mathrm{CDCl}_{3}$, $70.8\left(\mathrm{C}_{5} \mathrm{H}_{5}\right)$, n.o. Cp- $\mathrm{C}_{\mathrm{i}}$ and Th-C2; ${ }^{11} \mathrm{~B}$ NMR $\left(\mathrm{CDCl}_{3}, 160.3 \mathrm{MHz}\right): \mathrm{\square}=50.8\left(\mathrm{w}_{1 / 2}=400 \mathrm{~Hz}\right)$. 
Reaction of FcThBBr with $\mathbf{T h}_{2} \mathrm{Hx}_{2}\left(\mathrm{SnMe}_{3}\right)_{2}$ : Synthesis of M-BFc. $\mathrm{Th}_{2} \mathrm{Hx}_{2}\left(\mathrm{SnMe}_{3}\right)_{2}(0.87 \mathrm{~g}$, $1.32 \mathrm{mmol}$ ) was dissolved in $10 \mathrm{ml}$ of $\mathrm{CH}_{2} \mathrm{Cl}_{2}$ and cooled to $-35^{\circ} \mathrm{C}$ under stirring. A solution of FcThBBr $(0.95 \mathrm{~g}, 2.64 \mathrm{mmol})$ in $10 \mathrm{ml}$ of $\mathrm{CH}_{2} \mathrm{Cl}_{2}$ was added slowly under stirring. The reaction mixture was allowed to warm up slowly and left stirring at room temperature for $12 \mathrm{~h}$. All volatile components were removed under high vacuum and the crude product was heated to $60{ }^{\circ} \mathrm{C}$ for $6 \mathrm{~h}$ to remove trimethyltin bromide. The dark red crude material was recrystallized several times from hot hexanes. Isolated yield: $0.55 \mathrm{~g}(47 \%)$. For M-BFc: ${ }^{1} \mathrm{H} \mathrm{NMR}\left(\mathrm{CDCl}_{3}, 499.893 \mathrm{MHz}\right): \mathrm{\square}=7.84$ $\left(\mathrm{d},{ }^{3} \mathrm{~J}=4.5 \mathrm{~Hz}, 2 \mathrm{H}, \mathrm{Th}-\mathrm{H} 3 / 5\right), 7.80\left(\mathrm{~d},{ }^{3} \mathrm{~J}=3.5 \mathrm{~Hz}, 2 \mathrm{H}, \mathrm{Th}-\mathrm{H} 3 / 5\right), 7.62\left(\mathrm{~s}, 2 \mathrm{H}, \mathrm{Hx}_{2} \mathrm{Th}_{2}-\mathrm{H} 4,4^{\prime}\right), 7.34$ $\left(\mathrm{dd},{ }^{3} \mathrm{~J}=3.5 / 4.5 \mathrm{~Hz}, 2 \mathrm{H}\right.$, Th-H4), $4.88\left(\mathrm{pst},{ }^{3 / 4} \mathrm{~J}=2.0 \mathrm{~Hz}, 4 \mathrm{H}, \mathrm{Cp}-\mathrm{H}\right), 4.81\left(\mathrm{pst},{ }^{3 / 4} \mathrm{~J}=2.0 \mathrm{~Hz}, 4 \mathrm{H}\right.$, Cp-H), 4.20 (s, $\left.10 \mathrm{H}, \mathrm{C}_{5} \mathrm{H}_{5}\right), 2.74\left(\mathrm{t},{ }^{3} \mathrm{~J}=7.5 \mathrm{~Hz}, 4 \mathrm{H}, \mathrm{Th}-\mathrm{CH}_{2}\right), 1.69$ (m, 4H, hexyl-H), 1.40-1.20 $\left(\mathrm{m}, 12 \mathrm{H}\right.$, hexyl-H), $0.88\left(\mathrm{t},{ }^{3} \mathrm{~J}=7.0 \mathrm{~Hz}, 6 \mathrm{H}\right.$, hexyl-H) ${ }^{13} \mathrm{C} \mathrm{NMR}\left(\mathrm{CDCl}_{3}, 125.7 \mathrm{MHz}\right): \mathrm{C}=145.2$, $145.1\left(\mathrm{Hx}_{2} \mathrm{Th}_{2}-\mathrm{C} 5,5\right.$, Th-C2), $143.3\left(\mathrm{Hx}_{2} \mathrm{Th}_{2}\right), 141.8\left(\mathrm{Hx}_{2} \mathrm{Th}_{2}\right), 139.0$ (Th-C3), $138.5\left(\mathrm{Hx}_{2} \mathrm{Th}_{2}\right)$, 133.5 (Th-C4), 128.4 (Th-C5), 78.2, 75.674 .0 (Cp-C), $70.1\left(\mathrm{C}_{5} \mathrm{H}_{5}\right), 31.9$, 31.3, 29.5, 29.3, 22.9, 14.3 (hexyl-C); ${ }^{11} \mathrm{~B}$ NMR $\left(\mathrm{CDCl}_{3}, 160.3 \mathrm{MHz}\right): \mathrm{\square}=52.4\left(\mathrm{w}_{1 / 2}=2600 \mathrm{~Hz}\right)$; UV-Vis $\left(\mathrm{CH}_{2} \mathrm{Cl}_{2}\right)$ : $\square_{\max }$ $=307 \mathrm{~nm}(\square=27200), 350 \mathrm{~nm}(\square=21990), 493 \mathrm{~nm}(\square=3660)$; MALDI-TOF-TOF MS (benzo-apyrene): calcd 890.56, observed (positive mode) 890.21.

Reaction of ${ }^{\mathrm{i}} \mathrm{PrPhSnMe}_{3}$ with $\mathrm{ThBBr}_{2}$ : Synthesis of ( $\left.{ }^{(} \mathbf{P r P h}\right) \mathbf{T h B B r} . \mathrm{ThBBr}_{2}(2.00 \mathrm{~g}, 7.88$ $\mathrm{mmol})$ in $10 \mathrm{ml}$ of toluene was cooled to $-35^{\circ} \mathrm{C}$. A solution of ${ }^{\mathrm{i}} \mathrm{PrPhSnMe}_{3}(2.00 \mathrm{~g}, 7.17 \mathrm{mmol})$ in $10 \mathrm{ml}$ of toluene was added slowly under stirring. The reaction mixture was allowed to warm up slowly and left stirring at room temperature for $12 \mathrm{~h}$. All volatile components were removed under high vacuum, and the crude product was heated to $60^{\circ} \mathrm{C}$ for $6 \mathrm{~h}$ to remove trimethyltin bromide. The colorless crude material was $>97 \%$ pure (confirmed by ${ }^{1} \mathrm{H}$ NMR). Isolated yield: $2.0 \mathrm{~g}$ (96\%). For ('PrPh)ThBBr: ${ }^{1} \mathrm{H}$ NMR $\left(\mathrm{CDCl}_{3}, 499.893 \mathrm{MHz}\right)$ : $\square=8.08-8.00$ (m, 4H, Th-H3,5 and $\left.\mathrm{Ph}-\mathrm{H}_{o}\right), 7.40-7.35\left(\mathrm{~m}, 3 \mathrm{H}\right.$, Th-H3 and $\left.\mathrm{Ph}-\mathrm{H}_{m}\right), 3.00$ (heptet, $\left.{ }^{3} \mathrm{~J}=7.0 \mathrm{~Hz}, 1 \mathrm{H}, \mathrm{CH}\left[\mathrm{CH}_{3}\right]_{2}\right), 1.31\left(\mathrm{~d},{ }^{3} \mathbf{J}\right.$ $\left.=7.0 \mathrm{~Hz}, 6 \mathrm{H}, \mathrm{CH}\left[\mathrm{CH}_{3}\right]_{2}\right) ;{ }^{13} \mathrm{C} \mathrm{NMR}\left(\mathrm{CDCl}_{3}, 125.7 \mathrm{MHz}\right): \mathrm{C}=154.6\left(\mathrm{Ph}-\mathrm{C}_{p}\right), 143.5(\mathrm{Th}-\mathrm{C} 3), 139.6$ (Th-C5), $137.3\left(\mathrm{Ph}-\mathrm{C}_{o}\right), 129.7$ (Th-C4), $126.5\left(\mathrm{Ph}-\mathrm{C}_{m}\right), 34.6\left(\mathrm{CH}\left[\mathrm{CH}_{3}\right]_{2}\right), 23.9\left(\mathrm{CH}\left[\mathrm{CH}_{3}\right]_{2}\right)$, n.o. Th-C2, Ph-C $\mathrm{C}_{\mathrm{i}} ;{ }^{11} \mathrm{~B}$ NMR $\left(\mathrm{CDCl}_{3}, 160.3 \mathrm{MHz}\right): \mathrm{\square}=56.8\left(\mathrm{w}_{1 / 2}=480 \mathrm{~Hz}\right)$.

Reaction of ( $\left.{ }^{\mathrm{P} P P h}\right) \mathrm{ThBBr}$ with $\mathbf{T h}_{2} \mathrm{Hx}_{2}\left(\mathrm{SnMe}_{3}\right)_{2}$ : Synthesis of M-BPh. $\mathrm{Th}_{2} \mathrm{Hx}_{2}\left(\mathrm{SnMe}_{3}\right)_{2}(1.13$ $\mathrm{g}, 1.7 \mathrm{mmol}$ ) was dissolved in $10 \mathrm{ml}$ of $\mathrm{CH}_{2} \mathrm{Cl}_{2}$ and cooled to $-35^{\circ} \mathrm{C}$ under stirring. A solution of ( $\mathrm{PrPh}) \mathrm{ThBBr}(1.00 \mathrm{~g}, 3.4 \mathrm{mmol})$ in $10 \mathrm{ml}$ of $\mathrm{CH}_{2} \mathrm{Cl}_{2}$ was added slowly under stirring. The reaction mixture was allowed to warm up slowly and left stirring at room temperature for $12 \mathrm{~h}$. All volatile components were removed under vacuum, and the crude product was kept under high vacuum at $60{ }^{\circ} \mathrm{C}$ for $6 \mathrm{~h}$ to remove trimethyltin bromide. For further purification the crude product was dissolved in $10 \mathrm{ml}$ of hexanes and converted into the pyridine adduct through treatment with an excess of pyridine $(0.50 \mathrm{~g}, 6.3 \mathrm{mmol})$ in $5 \mathrm{ml}$ of hexanes. The mixture was stirred for $3 \mathrm{~h}$ and all volatile components were removed under high vacuum. The white residue was redissolved in hot hexanes and the pyridine adduct was allowed to precipitate at $-35{ }^{\circ} \mathrm{C}$ over a period of $24 \mathrm{~h}$. A solution of the pyridine adduct $(0.90 \mathrm{~g}, 0.98 \mathrm{mmol})$ in hexanes was then treated with $\mathrm{BCl}_{3}(3 \mathrm{ml}$, $1 \mathrm{M}$ in hexanes, $3 \mathrm{mmol}$ ). The mixture was stirred for $1 \mathrm{~h}$ and then filtered through a fritted funnel All volatile components were removed under high vacuum leaving behind the product as a fluorescent green oil. Isolated yield: $0.69 \mathrm{~g}(53 \%)$. For M-BPh: ${ }^{1} \mathrm{H} \mathrm{NMR}\left(\mathrm{CDCl}_{3}, 499.893 \mathrm{MHz}\right)$ : $\mathrm{C}=7.98\left(\mathrm{~d},{ }^{3} \mathrm{~J}=4.5 \mathrm{~Hz}, 2 \mathrm{H}, \mathrm{Th}-\mathrm{H} 3 / 5\right), 7.85\left(\mathrm{~d},{ }^{3} \mathrm{~J}=3.5 \mathrm{~Hz}, 2 \mathrm{H}, \mathrm{Th}-\mathrm{H} 3 / 5\right), 7.78\left(\mathrm{~d},{ }^{3} \mathrm{~J}=8.0 \mathrm{~Hz}\right.$, $\left.4 \mathrm{H}, \mathrm{Ph}-\mathrm{H}_{o}\right), 7.69$ (s, 2H, $\left.\mathrm{Hx}_{2} \mathrm{Th}_{2}-\mathrm{H} 4,4^{\prime}\right), 7.4(\mathrm{nr}, 2 \mathrm{H}, \mathrm{Th}-\mathrm{H} 4), 7.36\left(\mathrm{~d},{ }^{3} \mathrm{~J}=8.0 \mathrm{~Hz}, 4 \mathrm{H}, \mathrm{Ph}-\mathrm{H}_{m}\right.$ ), 3.03 (heptet, $\left.{ }^{3} \mathrm{~J}=7.0 \mathrm{~Hz}, 2 \mathrm{H}, \mathrm{CH}\left[\mathrm{CH}_{3}\right]_{2}\right), 2.73\left(\mathrm{t},{ }^{3} \mathrm{~J}=8.0 \mathrm{~Hz}, 4 \mathrm{H}\right.$, Th- $\left.\mathrm{CH}_{2}\right), 1.66(\mathrm{~m}, 4 \mathrm{H}$, hexyl- 
$\mathrm{H}), 1.37\left(\mathrm{~d},{ }^{3} \mathrm{~J}=7.0 \mathrm{~Hz}, 12 \mathrm{H}, \mathrm{CH}\left[\mathrm{CH}_{3}\right]_{2}\right), 1.35-1.20\left(\mathrm{~m}, 12 \mathrm{H}\right.$, hexyl-H), $0.87\left(\mathrm{t},{ }^{3} \mathrm{~J}=7.0 \mathrm{~Hz}, 6 \mathrm{H}\right.$, $\left.\mathrm{CH}_{3}\right) ;{ }^{13} \mathrm{C}$ NMR $\left(\mathrm{CDCl}_{3}, 125.7 \mathrm{MHz}\right): \square=151.9\left(\mathrm{Ph}-\mathrm{C}_{p}\right), 145.3\left(\mathrm{Hx}_{2} \mathrm{Th}_{2}-\mathrm{C} 5,5\right.$ '), 145.1 (Th-C2), 144.5 (Th-C3), $144.4\left(\mathrm{Hx}_{2} \mathrm{Th}_{2}\right), 142.4,141.8\left(\mathrm{Hx}_{2} \mathrm{Th}_{2}\right), 140.7\left(\mathrm{Ph}-\mathrm{C}_{i}\right), 137.5\left(\mathrm{Ph}-\mathrm{C}_{o}\right), 137.1(\mathrm{Th}-$ C5), 129.1 (Th-C4), $125.7\left(\mathrm{Ph}-\mathrm{C}_{m}\right), 34.5\left(\mathrm{CH}\left[\mathrm{CH}_{3}\right]_{2}\right), 24.1\left(\mathrm{CH}\left[\mathrm{CH}_{3}\right]_{2}\right), 31.9,31.2,29.4,29.2$, 22.9, 14.3 (hexyl-C); ${ }^{11} \mathrm{~B}$ NMR $\left(\mathrm{CDCl}_{3}, 160.3 \mathrm{MHz}\right): \square=53.1\left(\mathrm{w}_{1 / 2}=2080 \mathrm{~Hz}\right) ; \mathrm{UV}-\mathrm{V}$ is $\left(\mathrm{CH}_{2} \mathrm{Cl}_{2}\right)$ : $\square_{\max }=361 \mathrm{~nm}(\square=31240), 314 \mathrm{~nm}(\square=14720) ; \square_{\mathrm{em}, \max }=458 \mathrm{~nm}, 482 \mathrm{~nm}(\mathrm{sh})\left(\square_{\mathrm{exc}}=360 \mathrm{~nm}\right)$; quantum yield $(\square)=0.19$; MALDI-TOF-TOF MS (benzo-a-pyrene): calcd 758.84, observed (negative mode) 760.63 .

Reaction of $\mathbf{T h S n M e}_{3}$ with $\mathrm{PfBBr}_{2}$ : Synthesis of PfThBBr: $\operatorname{PfBBr}_{2}(0.51 \mathrm{~g}, 1.51 \mathrm{mmol})$ was dissolved in $10 \mathrm{ml}$ of $\mathrm{CH}_{2} \mathrm{Cl}_{2}$ and cooled to $-35^{\circ} \mathrm{C}$ under stirring. A solution of $\mathrm{ThSnMe}_{3}(0.374 \mathrm{~g}$, $1.51 \mathrm{mmol}$ ) in $10 \mathrm{ml}$ of $\mathrm{CH}_{2} \mathrm{Cl}_{2}$ was added under stirring over a period of $30 \mathrm{~min}$. The reaction mixture was kept stirring for $30 \mathrm{~min}$ and used without further purification for the synthesis of M-BPf. For PfThBBr: ${ }^{1} \mathrm{H}$ NMR $\left(\mathrm{CDCl}_{3}, 499.893 \mathrm{MHz}\right)$ : $\square=8.22\left(\mathrm{~d},{ }^{3} \mathrm{~J}=5.0 \mathrm{~Hz}, 1 \mathrm{H}, \mathrm{Th}-\mathrm{H} 3 / 5\right)$, $7.95\left(\mathrm{~d},{ }^{3} \mathrm{~J}=3.5 \mathrm{~Hz}, 1 \mathrm{H}\right.$, Th-H3/5), $7.40\left(\mathrm{dd},{ }^{3} \mathrm{~J}=3.5 / 5.0 \mathrm{~Hz}, 1 \mathrm{H}, \mathrm{Hx}_{2} \mathrm{Th}_{2}-\mathrm{H} 4,4^{\prime}\right) ;{ }^{19} \mathrm{~F}$ NMR $(470.2$ $\mathrm{MHz}_{\mathrm{CDCl}}$ ): $\square=-130.9\left(\mathrm{~m}, 2 \mathrm{~F}, \mathrm{Pf}-\mathrm{F}_{\mathrm{o}}\right),-151.5\left(\mathrm{t}, 1 \mathrm{~F},{ }^{3} \mathrm{~J}_{\mathrm{FF}}=20 \mathrm{~Hz}, \mathrm{Pf}-\mathrm{F}_{\mathrm{p}}\right),-162.0\left(\mathrm{~m}, 2 \mathrm{~F}, \mathrm{Pf}-\mathrm{F}_{\mathrm{m}}\right)$; ${ }^{13} \mathrm{C} \mathrm{NMR}\left(\mathrm{CDCl}_{3}, 125.7 \mathrm{MHz}\right): \square=145.6\left(\mathrm{dm},{ }^{1} \mathrm{~J}_{\mathrm{CF}}=246 \mathrm{~Hz}, \mathrm{Pf}_{-} \mathrm{C}_{\mathrm{o}}\right), 145.3$ (Th-C3), 143.8 (ThC5), $142.7\left(\mathrm{dm},{ }^{1} \mathrm{~J}_{\mathrm{CF}}=251 \mathrm{~Hz}, \mathrm{Pf}_{-} \mathrm{C}_{\mathrm{p}}\right), 137.7\left(\mathrm{dm},{ }^{1} \mathrm{~J}_{\mathrm{CF}}=253 \mathrm{~Hz}, \mathrm{Pf}-\mathrm{C}_{\mathrm{m}}\right), 130.7$ (Th-C4), 113.8 (b, Pf-C $\left.\mathrm{C}_{\mathrm{i}}\right)$, n.o. Th-C2; ${ }^{11} \mathrm{~B}$ NMR $\left(\mathrm{CDCl}_{3}, 160.3 \mathrm{MHz}\right): \square=54.0\left(\mathrm{w}_{1 / 2}=320 \mathrm{~Hz}\right)$.

Reaction of PfThBBr with $\mathrm{Th}_{2} \mathrm{Hx}_{2}\left(\mathrm{SnMe}_{3}\right)_{2}$ : Synthesis of M-BPf: A solution of $\mathrm{Th}_{2} \mathrm{Hx}_{2}\left(\mathrm{SnMe}_{3}\right)_{2}(0.50 \mathrm{~g}, 0.76 \mathrm{mmol})$ in $10 \mathrm{ml}$ of $\mathrm{CH}_{2} \mathrm{Cl}_{2}$ was added dropwise to a solution of PfThBBr in $\mathrm{CH}_{2} \mathrm{Cl}_{2}$, which was prepared as described above. The reaction mixture was allowed to warm up slowly and left stirring at room temperature for $12 \mathrm{~h}$. All volatile components were removed under high vacuum, and the crude product was heated to $60{ }^{\circ} \mathrm{C}$ for $6 \mathrm{~h}$ to remove trimethyltin bromide. The crude product was obtained as yellow crystals upon recrystallization from hexanes at $-35{ }^{\circ} \mathrm{C}$. Isolated yield: $250 \mathrm{mg}$ (39 \%). For M-BPf: ${ }^{1} \mathrm{H}$ NMR $\left(\mathrm{CDCl}_{3}, 499.893\right.$ MHz): $\square=8.08\left(\mathrm{~d},{ }^{3} \mathrm{~J}=4.5 \mathrm{~Hz}, 2 \mathrm{H}\right.$, Th-H3/5), $7.87\left(\mathrm{~d},{ }^{3} \mathrm{~J}=3.5 \mathrm{~Hz}, 2 \mathrm{H}, \mathrm{Th}-\mathrm{H} 3 / 5\right), 7.69$ (s, 2H, $\left.\mathrm{Hx}_{2} \mathrm{Th}_{2}-\mathrm{H} 4,4^{\prime}\right), 7.37\left(\mathrm{dd},{ }^{3} \mathrm{~J}=3.5 / 4.5 \mathrm{~Hz}, 2 \mathrm{H}\right.$, Th-H4), $2.66\left(\mathrm{t},{ }^{3} \mathrm{~J}=7.5 \mathrm{~Hz}, 4 \mathrm{H}, \mathrm{Th}-\mathrm{CH}_{2}\right), 1.60(\mathrm{~m}$, $4 \mathrm{H}$, hexyl-H), 1.3-1.2 (m, 12H, hexyl-H), $0.85\left(\mathrm{t},{ }^{3} \mathrm{~J}=7.5 \mathrm{~Hz}, 6 \mathrm{H}\right.$, hexyl- $\left.\mathrm{CH}_{3}\right) ;{ }^{19} \mathrm{~F}$ NMR $(470.2$ $\mathrm{MHz}, \mathrm{CDCl}_{3}$ ): $\square=-131.01\left(\mathrm{~m}, 4 \mathrm{~F}, \mathrm{Pf}-\mathrm{F}_{\mathrm{o}}\right),-154.21\left(\mathrm{t}, 2 \mathrm{~F},{ }^{3} \mathrm{~J}_{\mathrm{FF}}=20 \mathrm{~Hz}, \mathrm{Pf}-\mathrm{F}_{\mathrm{p}}\right),-162.7$ (m, 4F, Pf$\left.\mathrm{F}_{\mathrm{m}}\right) ;{ }^{13} \mathrm{C} \mathrm{NMR}\left(\mathrm{CDCl}_{3}, 125.7 \mathrm{MHz}\right): \square=146.1\left(\mathrm{dm},{ }^{1} \mathrm{~J}_{\mathrm{CF}}=245 \mathrm{~Hz}, \mathrm{Pf}-\mathrm{C}_{\mathrm{o}}\right), 141.6\left(\mathrm{dm},{ }^{1} \mathrm{~J}_{\mathrm{CF}}=234\right.$ $\left.\mathrm{Hz}, \mathrm{Pf}-\mathrm{C}_{\mathrm{p}}\right), 137.5\left(\mathrm{dm},{ }^{1} \mathrm{~J}_{\mathrm{CF}}=251 \mathrm{~Hz}, \mathrm{Pf}-\mathrm{C}_{\mathrm{m}}\right), 115.2\left(\mathrm{t},{ }^{2} \mathrm{~J}_{\mathrm{CF}}=27 \mathrm{~Hz}, \mathrm{Pf}-\mathrm{C}_{\mathrm{i}}\right), 145.7\left(\mathrm{Hx}_{2} \mathrm{Th}_{2}\right), 145.6$ $\left(\mathrm{Hx}_{2} \mathrm{Th}_{2}\right), 144.1$ (Th-C3), 142.7 and $142.5\left(\mathrm{Hx}_{2} \mathrm{Th}_{2}-\mathrm{C} 5,5\right.$ ' and Th-C2), 140.3 (Th-C5), 129.9 (ThC4), 31.7, 31.0, 29.3, 29.1, 22.8, 14.2 (hexyl-C); ${ }^{11} \mathrm{~B}$ NMR $\left(\mathrm{CDCl}_{3}, 160.3 \mathrm{MHz}\right): \square=49.2\left(\mathrm{w}_{1 / 2}=\right.$ $1600 \mathrm{~Hz})$; UV-Vis $\left(\mathrm{CH}_{2} \mathrm{Cl}_{2}\right): \square_{\max }=376 \mathrm{~nm}(\square=35750), 304 \mathrm{~nm}(\square=24000)$; fluorescence $\left(\mathrm{CH}_{2} \mathrm{Cl}_{2}\right): \square_{\mathrm{em}, \max }=478 \mathrm{~nm}, 508 \mathrm{~nm}(\mathrm{sh})\left(\square_{\mathrm{exc}}=400 \mathrm{~nm}\right), \square=0.18$; MALDI-TOF-TOF MS (benzoa-pyrene): calcd 854.64, observed (negative mode) 856.80 .

\section{References:}

[1] Fouad, S.; Collard, D. M. Adv. Mater. 2003, 15, 81.

[2] Sundararaman, A.; Jäkle, F. J. Organomet. Chem. 2003, 681, 134-142.

[3] Qin, Y.; Cheng, G.; Achara, O.; Parab, K.; Jäkle, F. Macromolecules 2004, 37, 7123-7131.

[4] Renk, T.; Ruf, W.; Siebert, W. J. Organomet. Chem. 1976, 120, 1-25.

[5] See also: Roesler, R.; Har, B. J. N.; Piers, W. E. Organometallics 2002, 21, 4300-4302. 
Figure S1. ${ }^{1}$ H NMR spectra for polymers PTh-BAr. a) Spectrum of PTh-BFc; b) spectrum of PTh-BPh; c) spectrum of PTh-BPf; the signals at ca. $7.6 \mathrm{ppm}$ are likely due to cyclic species, which were detected by MALDI-TOF-TOF measurements.

a)

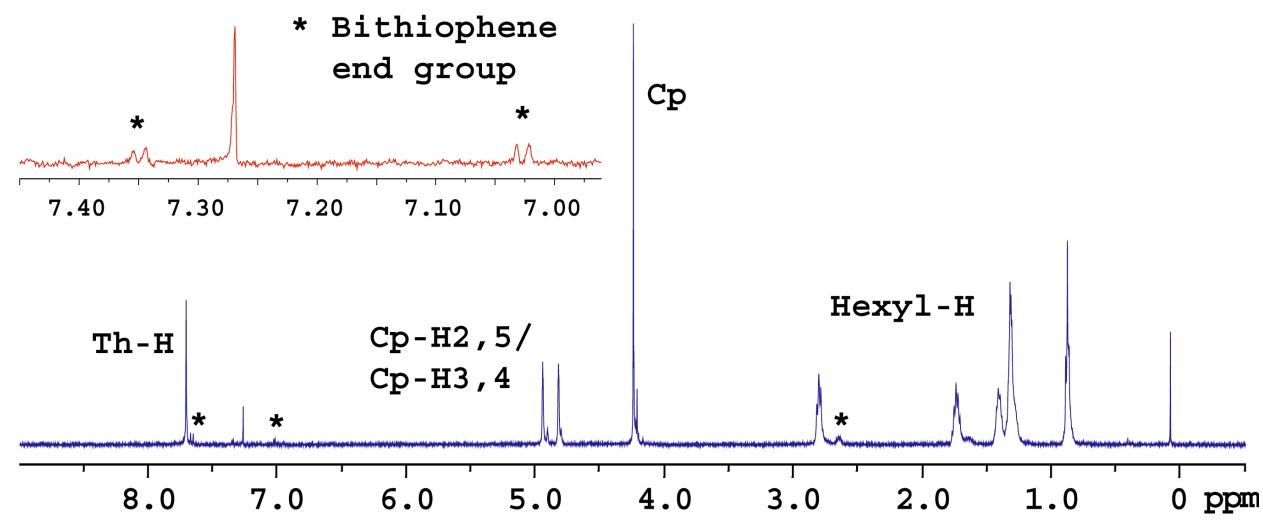

b)

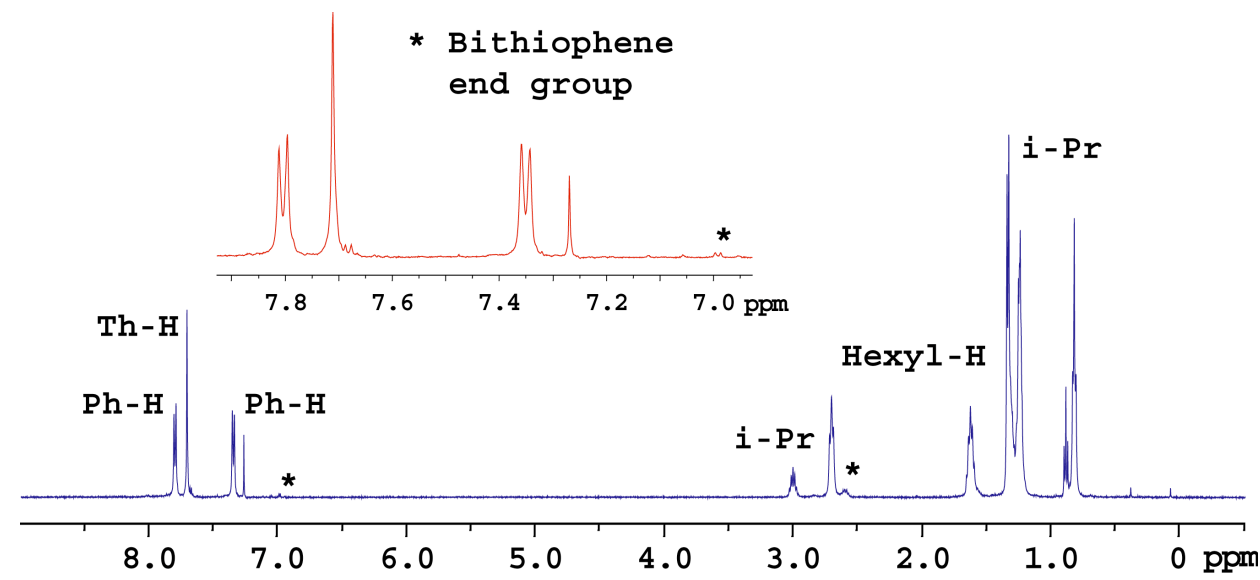

c)

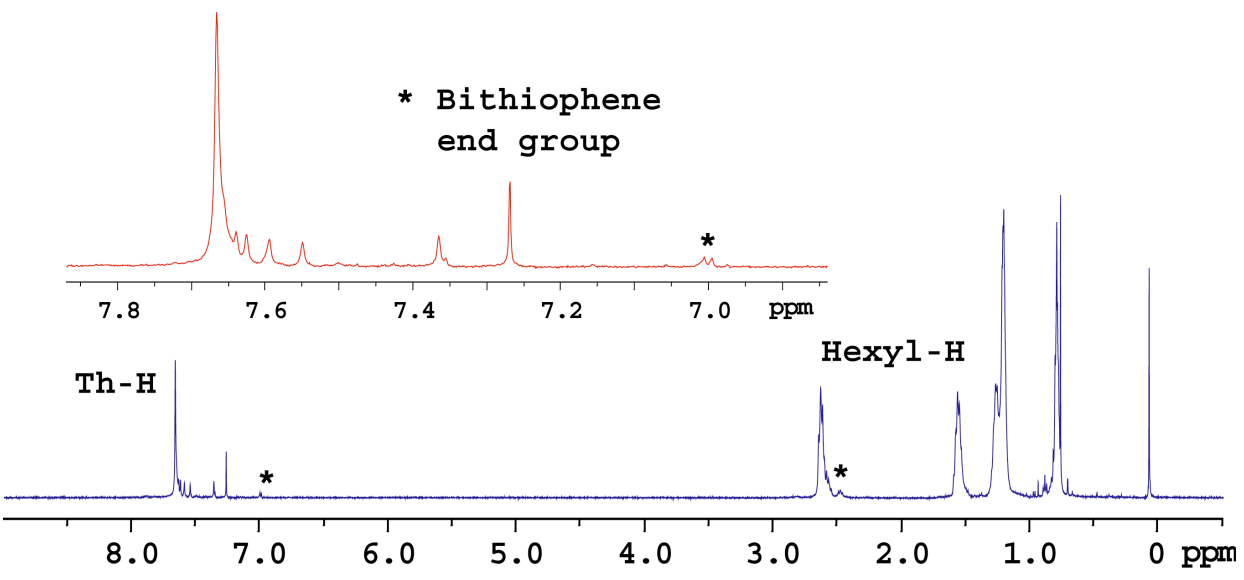


Figure S2. MALDI-TOF-TOF spectra for polymers PTh-BAr. a) Spectrum of PTh-BFc, $\mathrm{H}-\left\{\mathrm{C}_{20} \mathrm{H}_{28} \mathrm{~S}_{2} \mathrm{~B}\left(\mathrm{C}_{10} \mathrm{H}_{9} \mathrm{Fe}\right)\right\}_{\mathrm{n}}-\mathrm{C}_{20} \mathrm{H}_{29} \mathrm{~S}_{2}$, in positive mode; b) spectrum of $\mathbf{P} \mathbf{T h}-\mathbf{B} \mathbf{P h}$, $\mathrm{H}-\left\{\mathrm{C}_{20} \mathrm{H}_{28} \mathrm{~S}{ }_{2} \mathrm{~B}\left(\mathrm{C}_{9} \mathrm{H}_{11}\right)\right\}_{\mathrm{n}}-\mathrm{C}_{20} \mathrm{H}_{29} \mathrm{~S}_{2}$, in negative mode; the series of smaller peaks likely corresponds to species $\left(\mathrm{C}_{9} \mathrm{H}_{11}\right)_{2} \mathrm{~B}-\left\{\mathrm{C}_{20} \mathrm{H}_{28} \mathrm{~S}_{2}-\mathrm{B}\left(\mathrm{C}_{9} \mathrm{H}_{11}\right)\right\}_{\mathrm{n}}-\mathrm{C}_{9} \mathrm{H}_{11}$ as indicated by comparison with calculated molecular ion peaks; c) spectrum of PTh-BPf, $\mathrm{H}-\left\{\mathrm{C}_{20} \mathrm{H}_{28} \mathrm{~S}_{2} \mathrm{~B}\left(\mathrm{C}_{6} \mathrm{~F}_{5}\right)\right\}_{n}-\mathrm{C}_{20} \mathrm{H}_{29} \mathrm{~S}_{2}$, in negative mode; the series of smaller peaks corresponds to cyclic species $\left\{\mathrm{C}_{20} \mathrm{H}_{28} \mathrm{~S}_{2}-\mathrm{B}\left(\mathrm{C}_{6} \mathrm{~F}_{5}\right)\right\}_{\mathrm{n}}$ as indicated by comparison with calculated molecular ion peaks.

a)

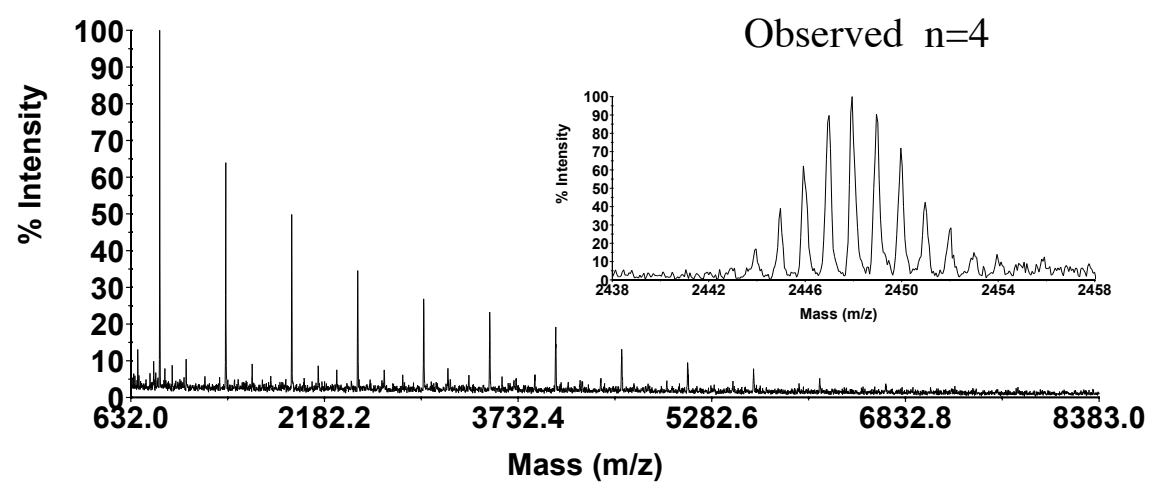

Calculated pattern $n=4$

b)

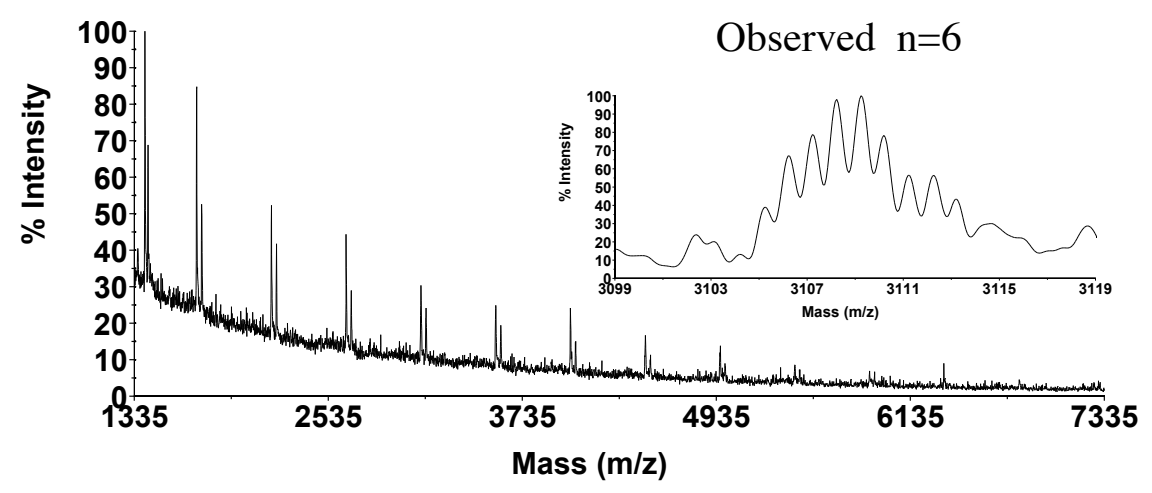

Calculated pattern $n=6$

c)

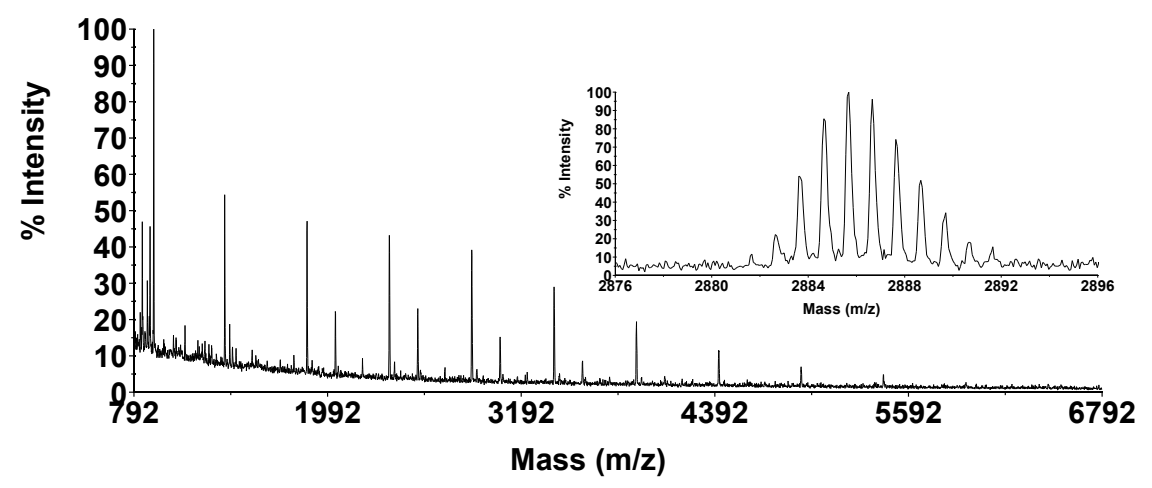

Calculated pattern $n=5$

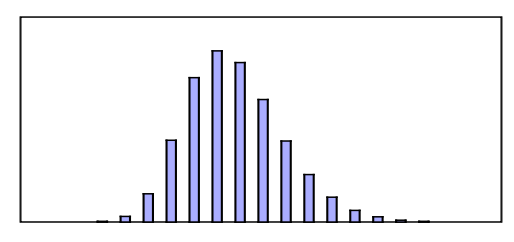


Figure S3. MALDI-TOF-TOF spectra for model compounds M-BAr. a) Spectrum of M-BFc in positive and negative mode; b) spectrum of M-BPh in negative mode; c) spectrum of PTh-BPf in negative mode.

a)

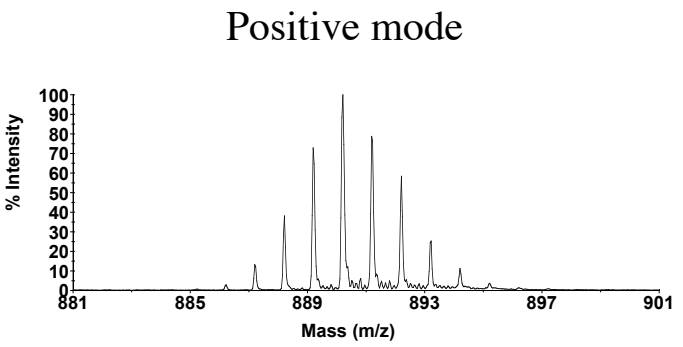

Negative mode (loss of $\mathrm{CpFe}^{+}$)

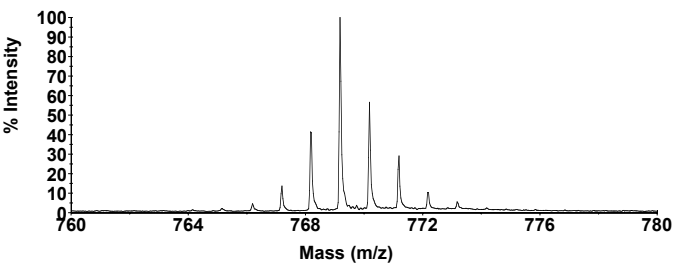

b)

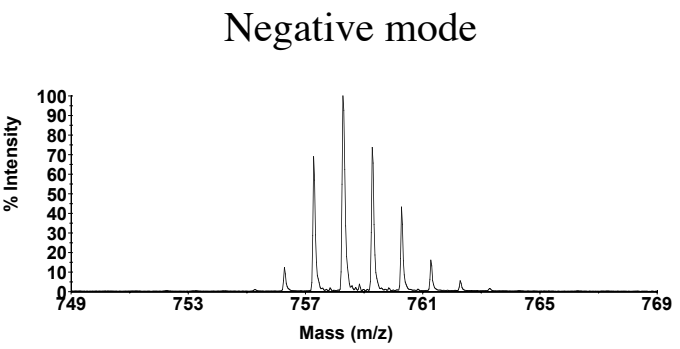

c)

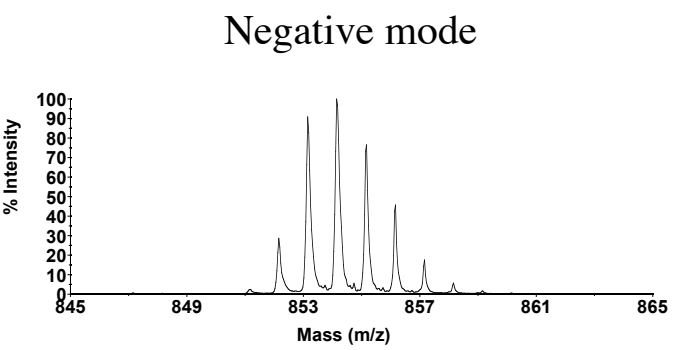

Calculated pattern for $\mathrm{C}_{48} \mathrm{H}_{52} \mathrm{~B}_{2} \mathrm{Fe}_{2} \mathrm{~S}_{4}$

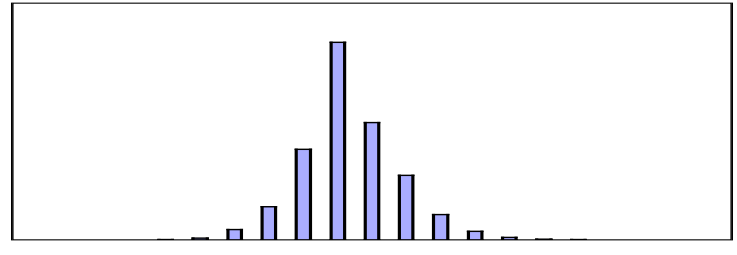

Calculated pattern for $\mathrm{C}_{43} \mathrm{H}_{47} \mathrm{~B}_{2} \mathrm{FeS} \mathrm{S}_{4}$

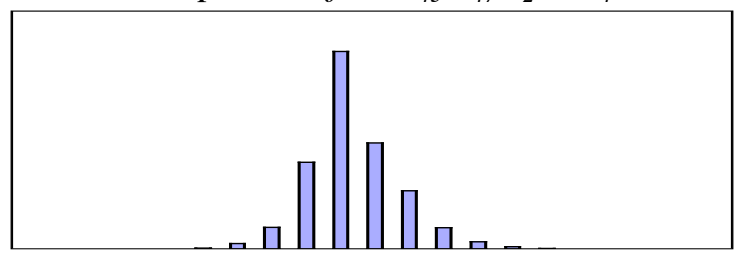

Calculated pattern for $\mathrm{C}_{46} \mathrm{H}_{56} \mathrm{~B}_{2} \mathrm{~S}_{4}$

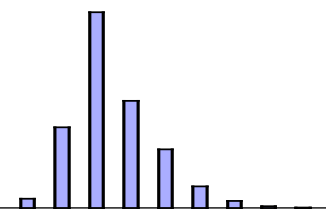

Calculated pattern for $\mathrm{C}_{40} \mathrm{H}_{34} B_{2} F_{10} \mathrm{~S}_{4}$

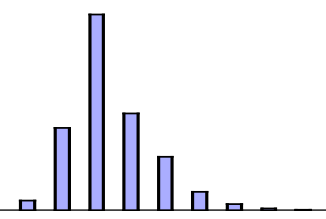


Figure S4. Absorption and emission spectra for polymers PTh-BAr in comparison to the model compounds M-BAr. a) Absorption spectra of PTh-BFc and M-BFc; b) absorption and emission spectra of PTh-BPh and M-BPh; c) absorption and emission spectra of PTh-BPf and M-BPf.

a)

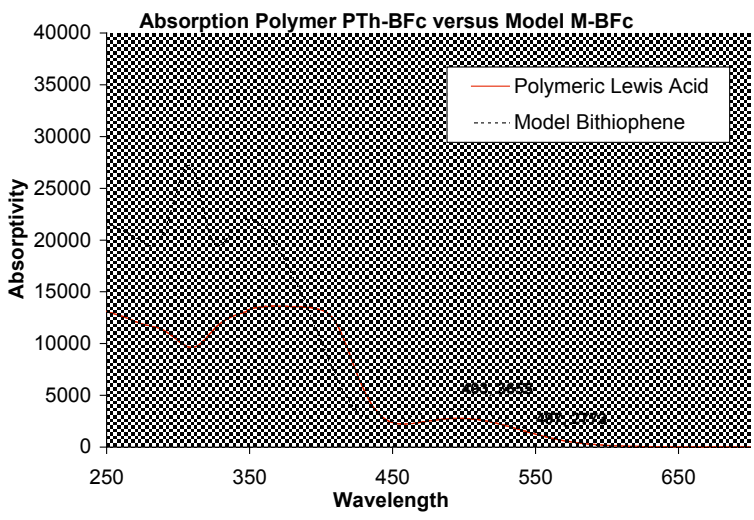

b)
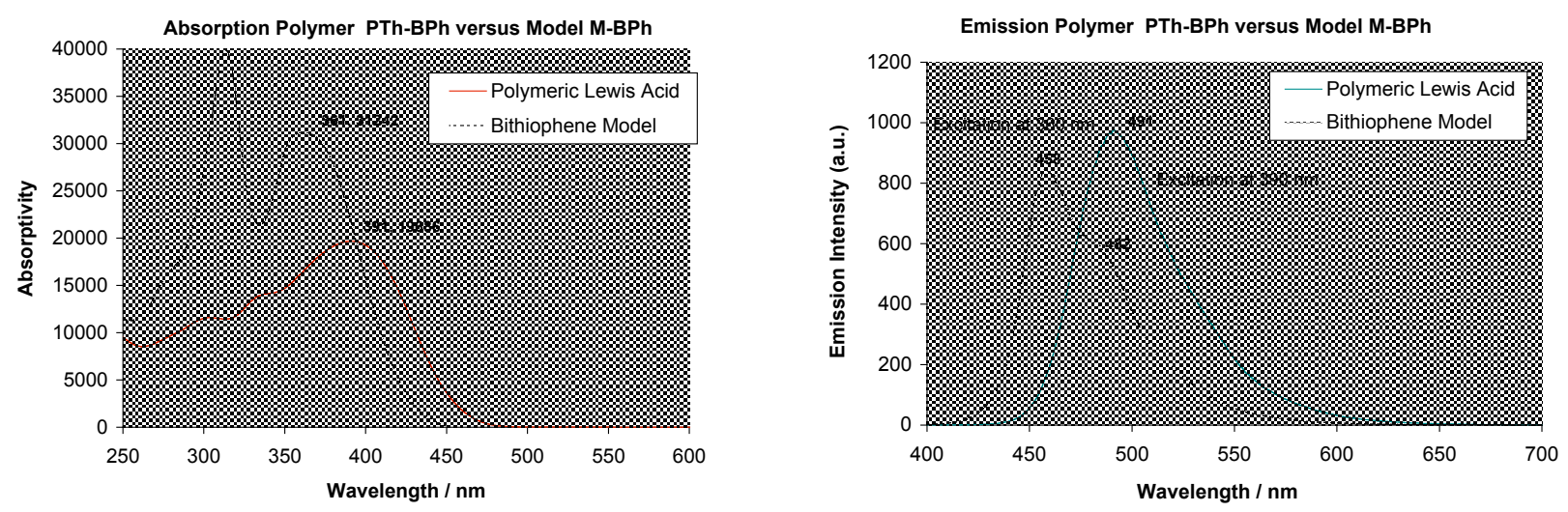

c)
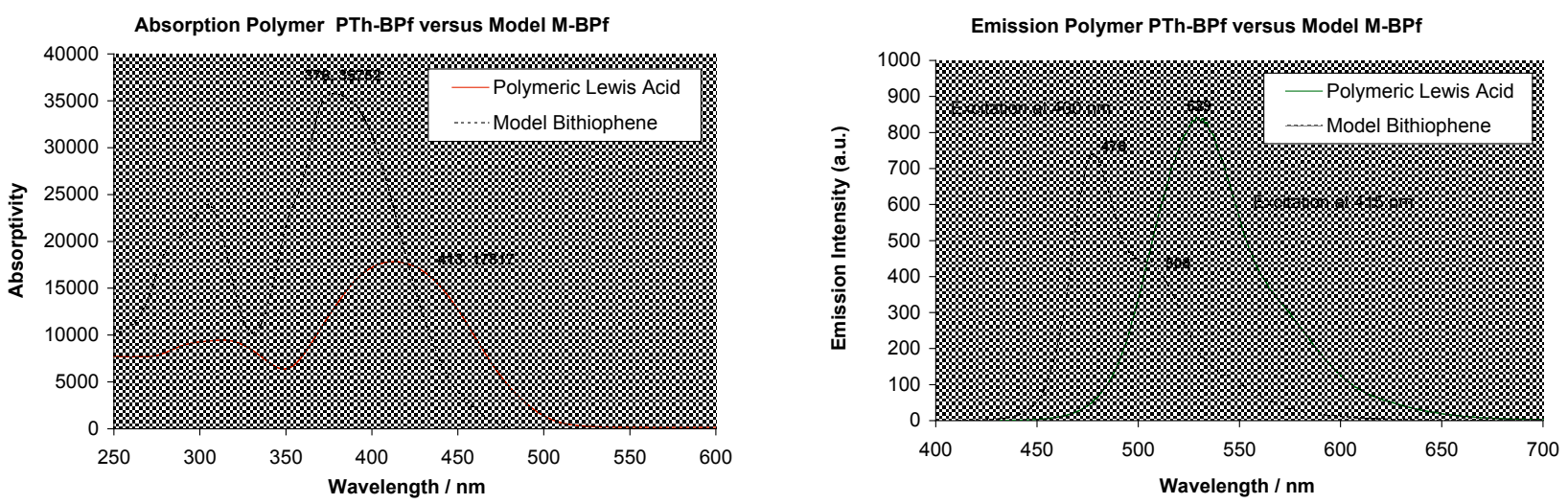
Figure S5. Pyridine titration of polymer solutions of PTh-BAr. a) titration of PTh-BFc $(2.1 \mathrm{x}$ $10^{-4} \mathrm{M}$ based on repeat units $)$ in $\mathrm{CH}_{2} \mathrm{Cl}_{2} ;$ b) titration of PTh-BPh $\left(6.8 \times 10^{-5} \mathrm{M}\right.$ based on repeat units) in $\mathrm{CH}_{2} \mathrm{Cl}_{2}$; c) titration of PTh-BPf $\left(4.6 \times 10^{-5} \mathrm{M}\right.$ based on repeat units) in $\mathrm{CH}_{2} \mathrm{Cl}_{2}$.

a)

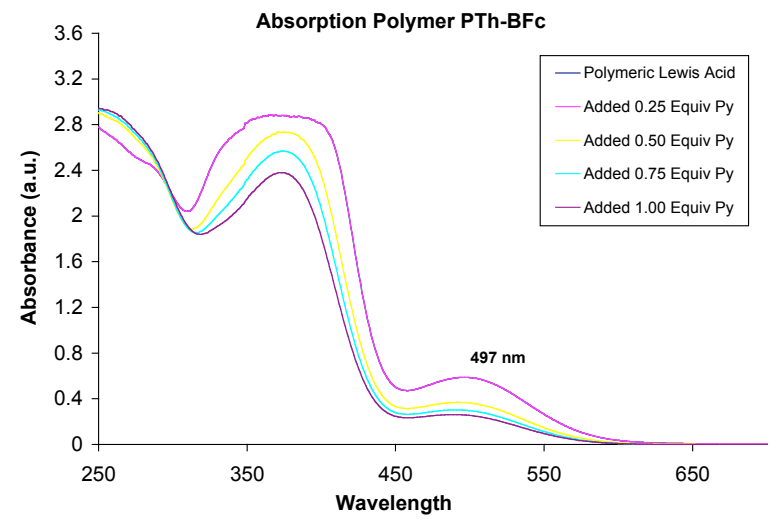

b)
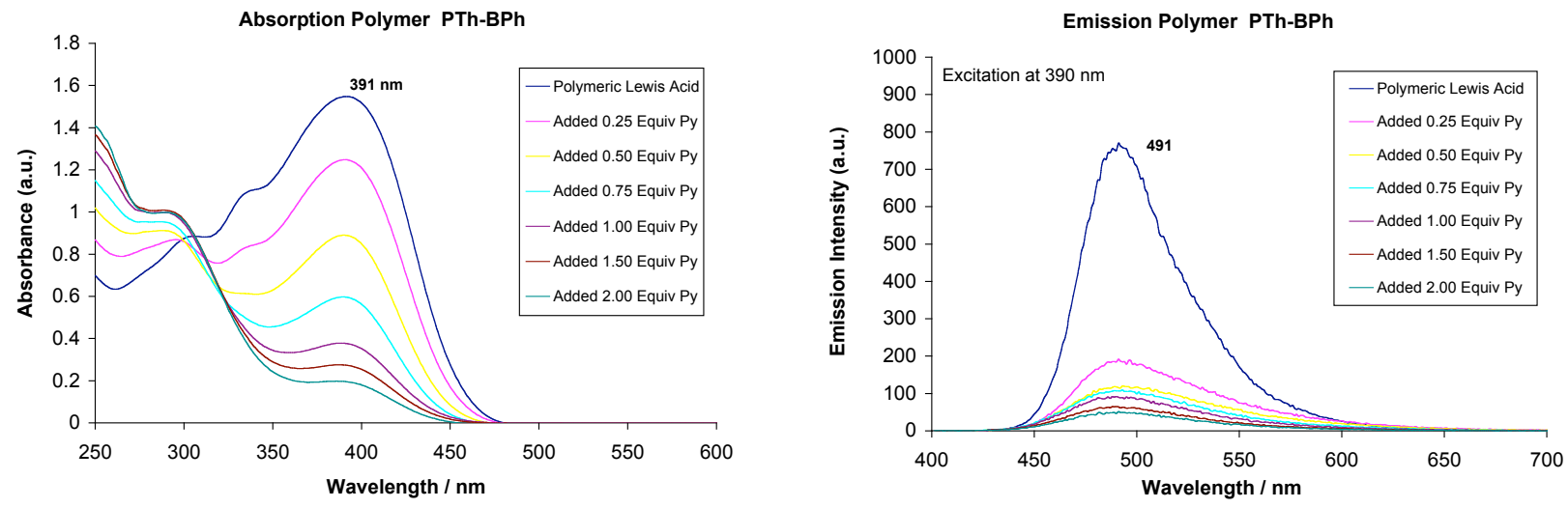

c)
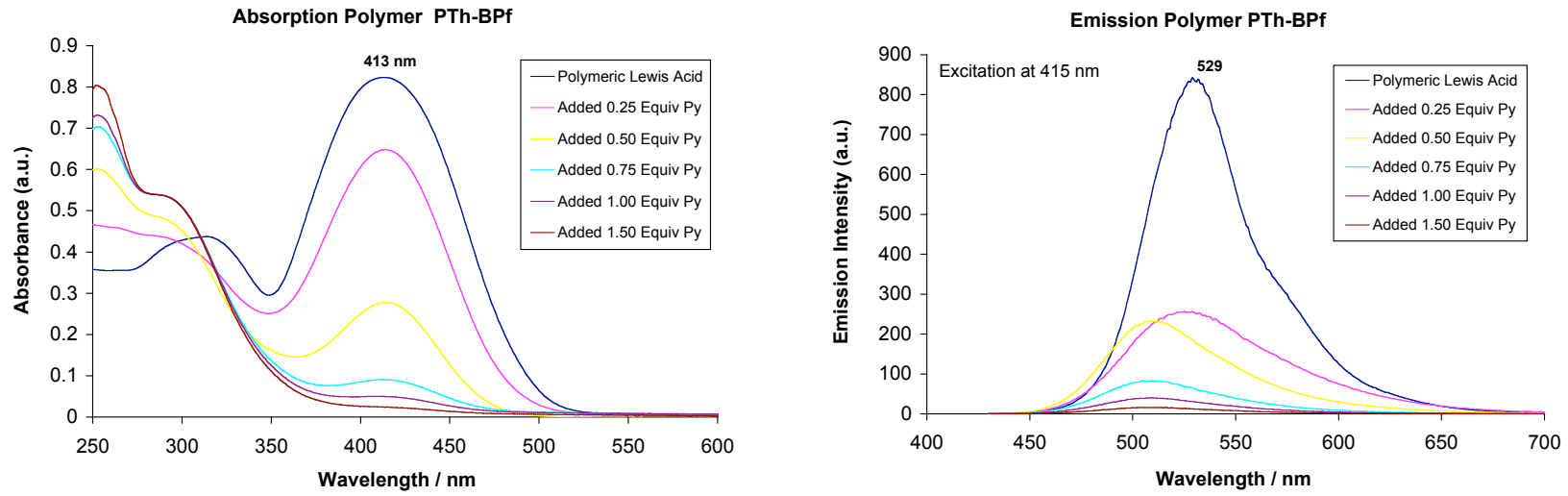
Figure S6. Binding selectivity studies. a) Treatment of PTh-BFc $\left(4.8 \times 10^{-5} \mathrm{M}\right.$ repeats in $\left.\mathrm{CH}_{2} \mathrm{Cl}_{2}\right)$ with equimolar amounts of different Lewis bases; b) treatment of PTh-BPh $\left(6.3 \times 10^{-5} \mathbf{M}\right.$ repeats in $\left.\mathrm{CH}_{2} \mathrm{Cl}_{2}\right)$ with equimolar amounts of different Lewis bases; c) treatment of PTh-BPf $\left(3.0 \times 10^{-5}\right.$ $\mathrm{M}$ repeats in $\mathrm{CH}_{2} \mathrm{Cl}_{2}$ ) with equimolar amounts of different Lewis bases.

a)

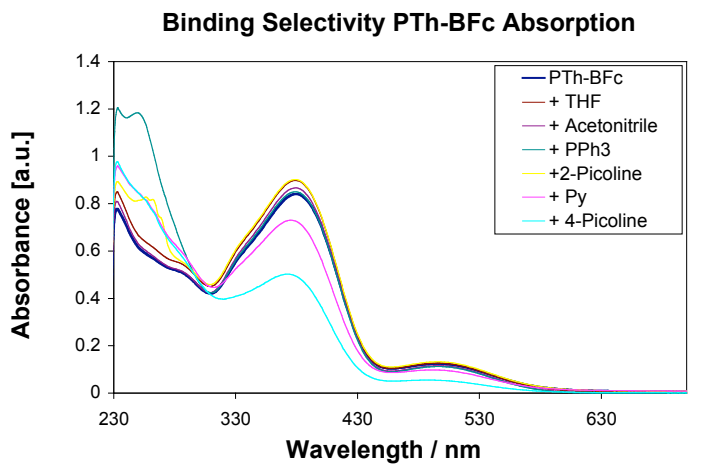

b)
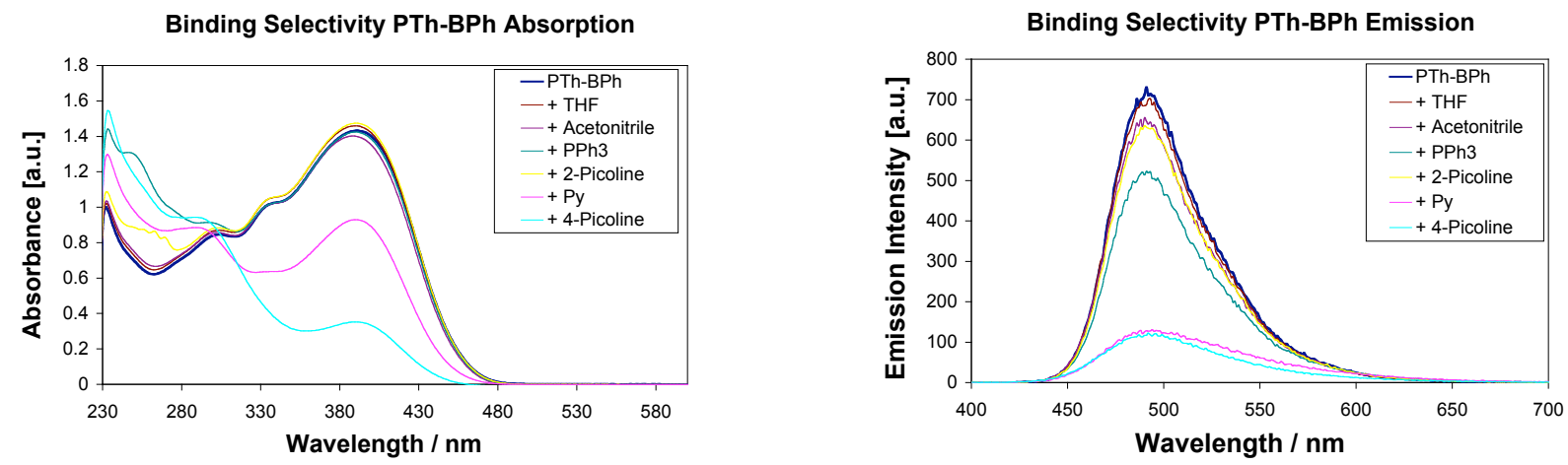

c)
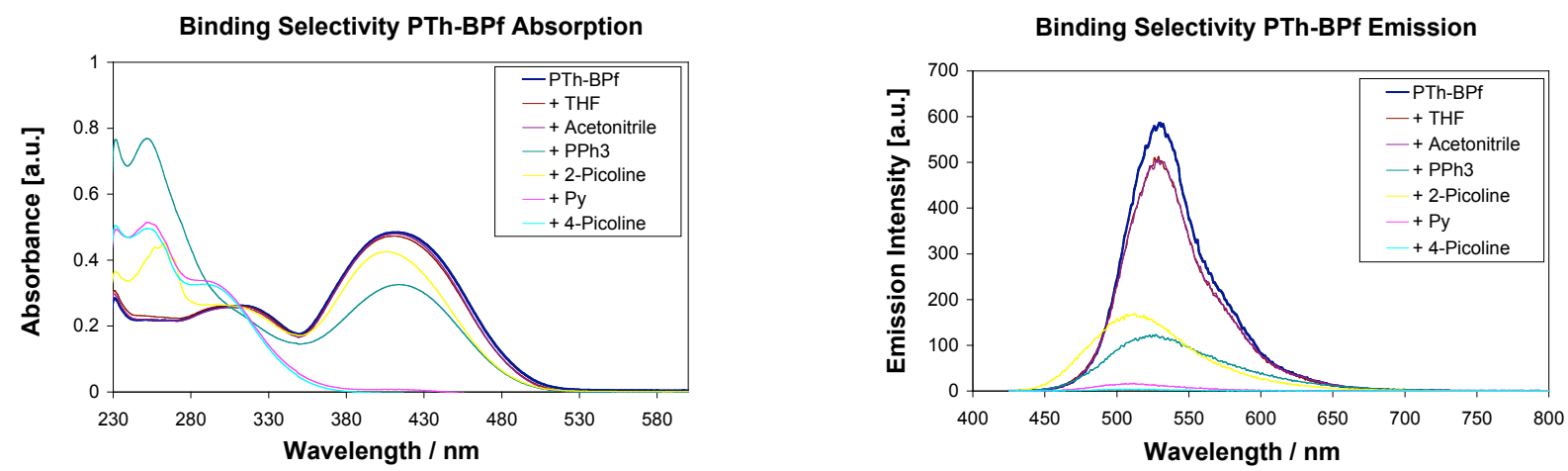
Figure S7. Evaluation of the negative cooperativity for pyridine binding to M-BPh. Spectral titration data were obtained using a $6.2710^{-5} \mathrm{M}$ solution of M-BPh and a 7.06 $10^{-4} \mathrm{M}$ solution of pyridine as the titrant $(25$ titration points between 0 and 10 molar equivalents of pyridine). Refinement was performed using the Hyperquad ${ }^{\mathrm{TM}}$ program suite with 37 different wavelengths between 245 and $425 \mathrm{~nm}$. A model for two independent consecutive binding events was used, where $\square_{11}$ and $\square_{12}$ were refined with $\square_{11}$

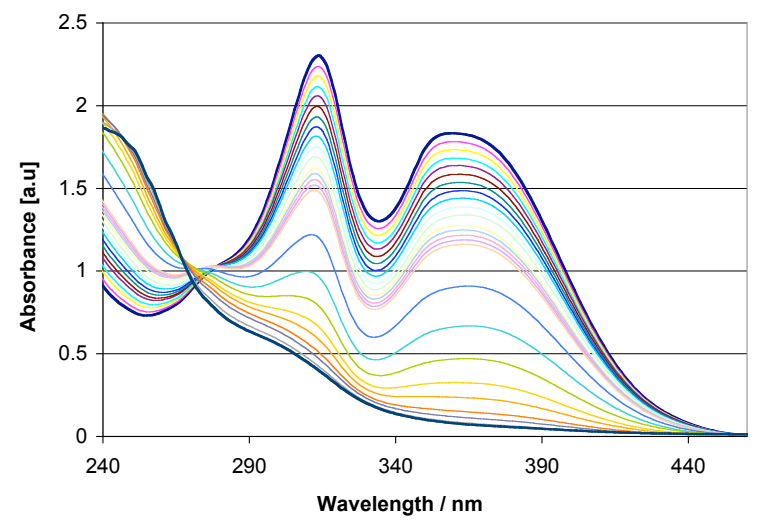
$=[\mathrm{M}-\mathrm{BPh} * \mathrm{Py}] /([\mathrm{M}-\mathrm{BPh}][\mathrm{Py}])$ and $\square_{12}=[\mathrm{M}-$ $\left.\mathrm{BPh} * \mathrm{Py}_{2}\right] /\left([\mathrm{M}-\mathrm{BPh}][\mathrm{Py}]^{2}\right)$.

Results:

$\lg \square_{11}$ refined to $6.42 \pm 0.08$

$\lg \square_{12}$ refined to $11.99 \pm 0.09$

$\mathrm{K}_{11}=7.0 \mathrm{~K}_{12}$ indicates negative cooperativity.

Figure S8. Stern Volmer plot for PTh-BPh in comparison to M-BPh. [PTh-BPh $]_{0}$ (based on repeat units $)=[\mathbf{M}-\mathbf{B P h}]_{0}=6.2710^{-5} \mathrm{M}$.

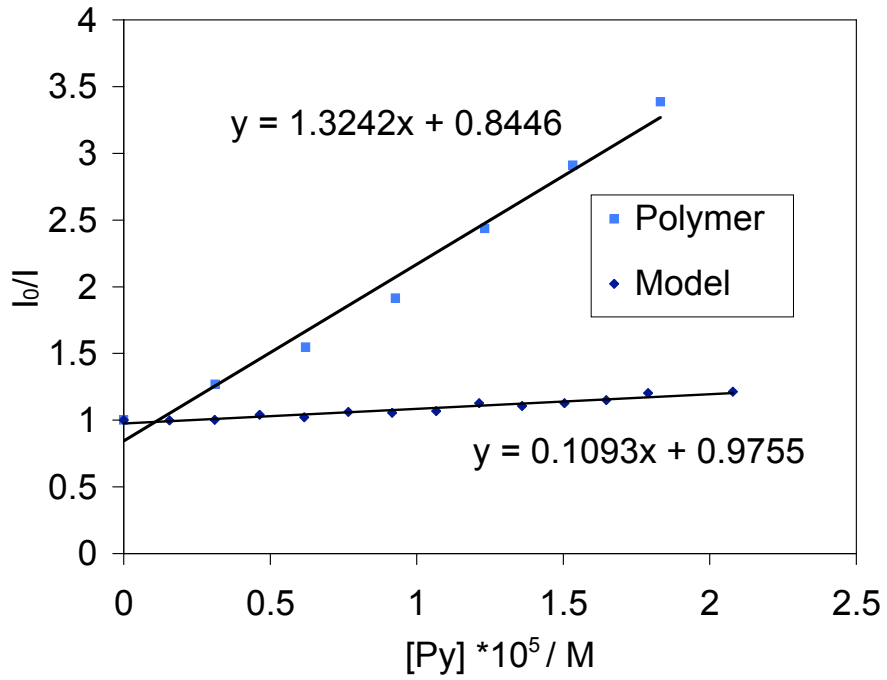

